\title{
Le corps des Ecritures digitales
}

Ce chapitre souhaite évaluer les conséquences de l'hypothèse générale exposée au chapitre 3 sur un cas particulier, les Ecritures bibliques. En effet si l'écriture digitale, en tant que nouvelle technologie, contribue au sein de ces dernières à développer un nouveau rapport du corps humain aux machines, il convient d'évaluer les conséquences de ce tournant épistémologique pour la notion de corpus textuel, qu'illustre avec netteté ce corpus millénaire désigné comme « les Ecritures », dans la tradition judéo-chrétienne.

Pour ce faire, le chapitre 4 va d'abord présenter un état des lieux de la recherche sur la Bible dans la culture digitale, en prêtant une attention particulière à ce qui est dit sur le statut du texte; il conclura à la nécessité de se pencher sur la notion de corps et de corpus (point 1). La seconde partie développera les notions de corpus textuel et corpus biblique, puis mettra en évidence le fait que la multimodalité est le lieu où les Ecritures digitales nouent leur lien à la corporéité (point 2). Nous aurons ainsi achevé notre parcours, dont les principales étapes sont récapitulées dans la conclusion de cet ouvrage.

\subsection{L'intérêt précoce des sciences bibliques pour l'informatique}

Comme signalé au chapitre 1 , les textes bibliques ont été présents très tôt dans les premiers projets en Humanities and Computing, via la confection d'une concordance biblique, en 1957, par le révérend John W. Ellison ${ }^{1}$, tandis que Roberto Busa s'occupait de Thomas d'Aquin et des rouleaux de la Mer Morte ${ }^{2}$. Puis en 1966, une première liste d'œuvres littéraires in Machine-Readable Form fut publiée ${ }^{3}$, suivie par des versions d'œuvres classiques déchiffrables par un ordinateur, notamment la Bible et toute l'œuvre de Platon ${ }^{4}$.

Certains biblistes se sont mis dès cette époque à réfléchir aux possibles conséquences de l'usage de l'ordinateur sur leur travail quotidien et leur

1 J. Solomon (éd.), Accessing Antiquity. The Computerization of Classical Studies, University of Arizona Press, 1993, p. 11 ; Jones, Roberto Busa, p. 100-101.

2 Jones, Roberto Busa, p. 13 ; voir chapitre 1, p. 30.

3 G. Carlson, «Literary Works in Machine-Readable Form. Computers and the Humanities 1 », Computer and the Humanities 1 (1967/3), p. 75-102.

4 Solomon (éd.), Accessing Antiquity, p. 136. 
champ, à commencer bien sûr par Kurt Aland pour l'Editio Critica Maior ${ }^{5}$, ou Bonifatius Fischer qui rédigeait en 1970 déjà un article sur le sujet ${ }^{6}$. Cette attention précoce des sciences bibliques à ce qui se joue dans le tournant informatique a été confirmé par Juan Garcés et Jan Heilmann dans un article rétrospectif en 2017.7 Ils soulignent notamment le « travail pionnier de A. Q. Morton (1978) et A. Kenny (1986) sur la comparaison stylométrique des lettres de Paul non contestées avec les lettres deutéro-pauliniennes $»^{8}$. Au début des années 9o, les sciences bibliques se sont retrouvées « de manière plus rapide et plus large » à avoir pris le train des humanités digitales, par rapport à d'autres domaines des sciences humaines ${ }^{9}$.

Les marques institutionnelles, publications et degrés académiques qui ont été signalés au chapitre 1 vont dans le sens de cette évaluation ${ }^{10}$, de même que le développement rapide d'outils pour les chercheurs et le grand public. Le numéro spécial Digital Humanities de la revue allemande Forum Exegese und Hochschuldidaktik offre un tour d'horizon assez complet du développement des humanités numériques pour les sciences bibliques, en soulignant notamment l'importance de Bibleworks, Accordance et Logos $^{11}$. On notera toutefois les grandes absences des projets francophones, pourtant déjà anciens comme la Bible informatique de l'Abbaye de Maredsous ou la Biblia Patristica - dès 1965 à

$5 \quad$ K. Aland, « Novi Testamenti Graeci Editio Maior Critica », NTS 16/2 (1970), p. 163-177.

6 B. Fischer, «The use of computer in New Testament studies, with special reference to textual criticism », JTS XXI (1970/2), p. 297-308.

7 J. Garcés, - J. Heilmann, « Digital Humanities und Exegese. Erträge, Potentiale, Grenzen und hochschuldidaktische Perspektiven », Forum Exegese und Hochschuldidaktik: Verstehen von Anfang an. Digital Humanities 2 (2017/2), p. 29-52.

8 Garcés, - Heilmann, « Digital Humanities », p. 32.

9 Garcés, - Heilmann, « Digital Humanities », p. 30 : « Es ist festzuhalten, dass die Digitalisierung und digitale Erforschung der für die Bibelwissenschaften (BW) relevanten Daten in vielerlei Hinsicht vergleichsmässig früher und umfangreicher als in anderen, verwandten geisteswissenchaftlichen Disziplinen an die sich Anfang der 1990er Jahre etablierenden und seitdem vielfach weiter Lehrer sowie Wissenschaftlerinnen und Wissenschaftler mittlerweile ausgereifte Texte in der digitalen Umgebung zu lesen und zu untersuchen ».

$10 \quad$ Voir chapitre 1, p. 16-18.

11 S. Fischer - T. Wagner, «Editorial », Forum Exegese und Hochschuldidaktik: Verstehen von Anfang an. Digital Humanities 2 (2017/2), p. 3-5 ; ici p. 3 : «In der biblischen Exegese wird bereits seit längerer Zeit mit digitalisierten und ausgezeichneten Texten gearbeitet, so dass morphologische Analysen und Konkordanzsuchen in systembasierten Programmen (BibleWorks, Accordance, Logos, etc.) bereits zu Standardverfahren gehören, die in Proseminaren vermittelt und von Studierenden mit Gewinn eingestezt wurden ». 
Strasbourg puis devenue BiblIndex à Lyon - ou d'ampleur comme La Bible en ses traditions mené par l'Ecole biblique de Jérusalem ${ }^{12}$.

Le constat d'une implication précoce des sciences bibliques dans la culture digitale se poursuit avec la monographie d'Heidi Campbell et de Stephen Garner, Networked Theology, publiée en 2016, qui adopte un point de vue proche de la pastorale, signalé d'entrée de volume :

En 2014, neuf pasteurs sur dix déclaraient qu'il est « théologiquement acceptable pour une église d'aider les personnes à cheminer dans la foi ou leurs expériences religieuses via internet». De manière générale, de nombreuses Eglises en Amérique considèrent qu'internet a muté d'un statut de produit de luxe à celui d'un outil essentiel pour le ministère ${ }^{13}$.

Campbell et Garner soulignent la collaboration entre l'American Bible Society et Microsoft ${ }^{14}$, ainsi que l'émergence d'applications bibliques dès les années 90, puis dans les années 2000 : on peine toutefois à comprendre les critères qu'ils ont retenus pour les applications bibliques qu'ils signalent, car elles sont de portées diverses et présentées sans critique ou évaluation ${ }^{15}$. De fait, tout au long de cette monographie orientée vers la pratique théologique des Eglises américaines, le statut du texte biblique, en tant qu'entité clairement définie et reconnue, n'est absolument pas mis en question par l'interaction avec la culture

Bible pastorale de Maredsous, <http://www.knowhowsphere.net/Main.aspx?BASEID= MARP> ; BiblIndex, <http://www.biblindex.mom.fr/fr/>; La Bible en ses traditions, $<$ https://scroll.bibletraditions.org/>.

Campbell - Garner, Networked Theology, p. 1 : « In 2014 nine out of ten pastors believed that it is "theologically acceptable for a church to provide faith assistance or religious experiences to people through the Internet". Overall, many churches in America viewed the internet as having moved from being a luxury to being essential tool for ministry ». Campbell - Garner, Networked Theology, p. 1.

15 Campbell - Garner, Networked Theology, p. 63: «Through the 199os, people from traditional and non-traditional religions experimented with creating new religious resources online. For example, Gospelcom (www.gospelcom.net) provided Christians with access to online Bible-study tools and various interactive devotional and fellowship groups. In the late 1990s, interreligious information hubs such as Beliefnet (www.beliefnet.org) emerged online, offering everything from thoughts for the day from the pope to inspirational screensavers and access to sacred texts. By the 2000 s religion online had become commonplace on the internet's landscape. Blogging platforms such as LivreJournal and Blogger.com allowed religious blogs and blog hubs to emerge, such as Christian Bloggy Moms (www.bloggymoms.com) hosting Christian mommy blogger pages and The Gospel Coalition (http://thegospelcoalition.org/blogs) offering Christians a common space to blog about their faith ». 
digitale ${ }^{16}$, et demeure désigné comme un texte écrit, a « written text » ${ }^{17}$, malgré la description de ses multiples usages fragmentés sur le Web. Le volume est porté par la conviction que le christianisme et la Bible pourront prendre le tournant numérique sans transformation fondamentale :

La Bible elle-même peut être vue comme une expression tangible, médiatique et technologique, en ce qu'elle est typiquement un artefact physique créé par l'humain (bien que d'autres représentations médiatiques soient possibles), et qui a été produit par une connaissance particulière. La forme et le contenu de la Bible, aussi bien que la forme et l'aspect de la communauté chrétienne, sont influencés par des environnements sociaux et culturels distincts. Si certaines personnes pourraient ne pas considérer le christianisme et la technologie comme profondément connectés à leur univers quotidien, la Bible offre un exemple utile de la manière dont le christianisme a pu négocier avec la technologie et les médias tout au long de son histoire ${ }^{18}$.

A lire cette déclaration de continuité médiatique de la Bible, on ne s'étonnera pas de ne pas trouver de questionnement sur le statut du texte religieux dans la culture digitale au sein d'un collectif dirigé trois ans auparavant par Campbell ${ }^{19}$. Cette conviction que la Bible peut traverser sans remise en question l'évolution des supports d'écriture se retrouve exprimée de diverses manières, plus ou moins explicites. A ce propos, il est particulièrement intéressant de lire en détail l'article de Juan Garcés et Jan Heilmann, qui marque une étape importante dans cet état de la recherche, comme il s'agit du premier article qui fait le tour d'horizon de l'exégèse néotestamentaire en regard des humanités digitales.

Tout comme chez Campbell et Garner, on y trouve l'affirmation d'une continuité de l'évolution de la technologie biblique, de la concordance établie par

16 Par exemple Campbell - Garner, Networked Theology, p. 67, 71, 76, 110 et 130.

17 Campbell - Garner, Networked Theology, p. 139.

18 Campbell - Garner, Networked Theology, p. $23:$ : The Bible itself can be seen as a tangible expression of technology and media in that it is typically a human-created physical artifact (though other mediated representations are possible) that has been produced by special knowledge. The Bible's form and content, as well as the form and shape of the Christian community, are influenced by distinct social and cultural environments. While people may not consider Christianity and technology deeply connected in their everyday world, the Bible provides a useful example of how Christianity has been negotiating with technology and media through out its history ».

19 Campbell (éd.), Digital Religion; il y est brièvement question de la Bible aux pages 108, 200, 201 et 216. 
Hugues de St-Cher en 1230 aux concordances électroniques ${ }^{20}$. Mais ces deux auteurs ouvrent également la porte à une réflexion sur le statut du texte, bien que de manière encore discrète. La partie de l'article qui est la plus importante pour notre sujet, «Handschriften, Textkritik und Edition », s'étend sur quatre pages ${ }^{21}$. Garcés et Heilmann s'y réfèrent essentiellement à l'Editio Critica Maior et aux travaux de l'INTF et ITSEE, largement soulignés également dans notre premier chapitre ${ }^{22}:$ «Dans la critique textuelle néotestamentaire, la méthode généalogique et basée sur la cohérence, développée par Gerd Mink et à la base de l'Editio Critica Maior (ECM) du Nouveau Testament grec, s'est imposée comme nouveau standard ${ }^{23}$. Il est à relever qu'une importante monographie introductive à cette méthodologie a été publiée en 2017 par Tommy Wasserman et Peter J. Gurry ${ }^{24}$. Une synthèse du tournant pris par l'ECM en matière de digitalisation des transcriptions des manuscrits a par ailleurs été présentée dans un article récent de H.A.G. Houghton ${ }^{25}$. Nous reviendrons à l'un et à l'autre.

Garcés et Heilmann complètent ensuite leur propos avec la présentation détaillée de la thèse de Stephen Carlson ${ }^{26}$, et les échos de cette dernière dans le projet ParaTextBib, mené par Martin Walraff à Münich ${ }^{27}$. Le lecteur ne peut bien sûr que se réjouir de ces compléments, mais reste tout de même surpris de ne voir dans ces pages aucune mention des autres éditions critiques du Nouveau Testament grec que j'ai signalées au chapitre 1, et qui questionnent par le simple fait de leur publication, respectivement en 2005, 2010 et $2017^{28}$. Pas de mention non plus chez Garcés et Heilmann des approches en critique

20 Garcés, - Heilmann, « Digital Humanities », p. 34.

21 Garcés, - Heilmann, « Digital Humanities », p. 39-42.

22 Voir chapitre 1, p. 35-36.

23 Garcés, - Heilmann, « Digital Humanities », p. 39: «In der neutestamentlichen Textkritik hat sich die von Gerd Mink entwickelte und der Editio Critica Maior des Novum Testamentum Graecum zugrunde liegenden "Kohärenzbasierte Genealogische Methode" (КВGM) als der neue Standard etabliert ».

24 T. Wasserman - P. J. Gurry, A New Approach to Textual Criticism: An Introduction to the Coherence-Based Genealogical Method (Resources for Biblical Study 80), sBL Press, 2017.

25 H.A.G. Houghton, « Electronic Transcriptions of New Testament Manuscripts and their Accuracy, Documentation and Publication », dans D. Hamidović - C. Clivaz - S. Bowen Savant (éd.), Ancient Manuscripts in Digital Culture (DBS 3), Brill, 2019, p. 133-153.

26 S. C. Carlson, The Text of Galatians and Its History (WUNT II/385), Mohr Siebeck, 2015.

27 ParaTextBib, <http://paratexbib.eu/>.

28 Pierpont - Robinson (éd.), The New Testament in the original Greek Byzantine Textform 2005, 2005; Version 2011: Pierpont - Robinson - Dodson (éd.), The New Testament in the Original Greek: Byzantine Textform 2018, 2018 ; Holmes (éd.), The sBL Greek New Testament, 2010 ; Jongkind - Head - Williams, The Tyndale House Greek New Testament (THGNT), 2017. Voir chapitre 1, p. 32. 
textuelle qui ont tenté d'appliquer informatiquement les méthodologies phylogénétiques, empruntées à la biologie ${ }^{29}$.

Les travaux portant sur l'édition critique digitale du point de vue des humanités numériques sont absents également, à commencer par le programme informatique Collate de Peter Robinson $(1994)^{30}$, beaucoup sollicité par la critique textuelle du Nouveau Testament ${ }^{31}$. Nos auteurs ne mentionnent pas non plus les importants travaux de la Text Encoding Initiative, par exemple le collectif de Lou Burnard et al. en 2006 $6^{32}$, ou les collectifs de Willard McCarty en 2010, de Daniel Apollon, Claire Bélisle et Philippe Régnier en 2014, sans oublier la récente monographie d'Elena Pierazzo ${ }^{33}$. Force est de noter que l'approche interdisciplinaire n'est pas prise en compte ici, alors que son apport est capital dans la discussion du statut du texte biblique dans la culture digitale, comme nous le verrons ${ }^{34}$.

En revanche, Garcés et Heilmann se risquent à une ouverture discrète, en deux étapes, en direction d'une plus grande flexibilité du rapport au texte biblique dans la culture digitale. En page 42, ils rappellent d'abord que les hypothèses de reconstruction historico-critique restent indispensables, unverzichtbar, et que la gestion du flux des données est à leur service, en tant que documentation sous-jacente ${ }^{35}$. Puis en page 44 , la formulation se fait un peu plus ouverte : «pour la recherche en histoire textuelle, ce n'est plus seulement la reconstruction d'une formulation précise qui est au centre des intérêts, mais les données qui ont conduit à la reconstruction, et les étapes d'analyse qui en

$29 \quad$ Voir point 1.3 ci-dessous.

30 P. Robinson, Collate : Interactive Collation of Large Textual Traditions, Version 2, Computer Program distributed by the Oxford University Centre for Humanities Computing, 1994. H.A.G. Houghton, «Electronic Transcriptions », p. 79. Il signale aussi cet article récent de Robinson : P. Robinson, « Some Principles for Making Collaborative Scholarly Editions in Digital Form », DHQ 11.2 2017, <http://www.digitalhumanities.org/dhq/vol/11/2/ooo293/ oo0293.html $>$.

32 L. Burnard et al. (éd.), Electronic Textual Editing, MLAA, 2006.

33 W. McCarty (éd.), Text and Genre in Reconstruction. Effects of Digitalization on Ideas, Behaviours, Products and Institutions (Digital Humanities series), OpenBook Publishers, 2010, <https://books.openedition.org/obp/630> ; D. Apollon - C. Bélisle - P. Régnier (éd.), Digital Critical Editions (Topics in the Digital Humanities), University of Illinois Press, 2014 ; E. Pierazzo, Digital Scholarly Editing. Theories, Models and Methods (Digital Research in the Humanities), Routledge Press, 2015.

34 Voir points 1.3 et 2.1 ci-dessous.

35 Garcés - Heilmann, «Digital Humanities », p. 42 : « Historisch-kritische Rekonstruktionen bleiben als Hypothesen über die historische Gestalt eines Textes für geschichtswissenschaftliches Arbeiten unverzichtbar. Es geht vielmehr darum, dass die erschlossenen Daten nicht mehr einer solchen Rekonstruktion lediglich als Dokumentation und Begründung der historisch-kritischen Entscheidungen unterworfen werden ». 
ont été tirées » ${ }^{36}$. Si Garcés et Heilmann restent ainsi prudents dans leur évaluation de la mise en données du texte biblique et ne prennent pas en compte la diversification des éditions du Nouveau Testament grec, c'est sans doute parce qu'ils conservent la conviction claire que le corpus de textes bibliques reste stable dans la culture digitale ${ }^{37}$. En ce sens, ils sont clairement inscrits dans la lignée présentée dans ce point 1.1, depuis le premier projet biblique par Ellison en 1957 .

Mais c'est ici que l'état de la recherche réserve la plus grande surprise : la première monographie consacrée à la Bible dans la culture digitale n'a été publiée qu'en 2017 par Jeffrey Siker ${ }^{38}$, soit soixante ans après qu'Ellison ait noué les premiers contacts entre Bible et informatique. La suite de ce premier point va s'appliquer à comprendre cet état de fait, en interrogeant notamment le statut du texte biblique dans la culture digitale, qui n'a pas paru problématique à la très grande majorité des chercheurs, jusqu'à récemment : notre hypothèse pour expliquer ces soixante années de décalage sera de dire qu'il fallait réaliser les enjeux de cette thématique pour y consacrer une monographie.

Comme l'indique le titre de l'ouvrage de Siker, Liquid Scripture, l'évaluation de la résistance du corpus biblique à la liquéfaction des discours écrits sur internet y est abordée frontalement. Le point 1.4 discutera ce livre et conclura le parcours du point 1 en soulignant la nécessité de revisiter la notion de corps, corpus textuel et corps des Ecritures (point 2). Auparavant, le point 1.2 va conduire l'analyse à partir des articles de Kurt Aland et Bonifatius Fischer, puis traverser le discours des critiques textuels du Nouveau Testament, en prêtant attention au statut du texte, abordé d'un point de vue interdisciplinaire en 1.3.

\subsection{Quand le statut du texte biblique n'est pas questionné}

L'article de Kurt Aland, qui présente en 1970 l'état des lieux sur « une nouvelle grande édition critique du Nouveau Testament », marque une étape capitale non seulement pour la critique textuelle du Nouveau Testament, mais aussi dans l'histoire de la rencontre entre ce corpus textuel et l'informatique ${ }^{39}$. De manière à la fois paradoxale et révélatrice, Kurt Aland n'aborde l'apport de l'ordinateur à cette nouvelle édition que dans les trois dernières pages de l'article, mais c'est pourtant un point clé, car c'est l'ordinateur qui va rendre possible

36 Garcés - Heilmann, « Digital Humanities », p. 44: « Für die textgeschichtliche Forschung ist nicht mehr nur die Rekonstruktion eines bestimmten Wortlauts von zentralem Interesse, sondern die zur Rekonstruktion führenden Daten und die analytischen Schritte, die daran vorgenommen wurden ».

37 Garcés, - Heilmann, « Digital Humanities », p. 37-38, notamment.

$38 \quad$ Siker, Liquid Scripture.

39 Aland, « Novi Testamenti Graeci ». 
cette édition, explique-t-il ${ }^{40}$. C'est ainsi qu'arrive l'ordinateur dans l'ensemble des sciences humaines de cette époque : avec le statut d'auxiliaire, mais prenant déjà plus que cette place qu'on veut lui accorder. Il se fait cheval de Troie de la transformation en cours.

Dans un paragraphe qui désormais fait date, Aland décrit la manière dont l'ordinateur va être sollicité dans cette nouvelle ECM, tablant notamment une «mise en ordre » des variantes des manuscrits ${ }^{41}$. Dans ces trois pages conclusives qui font place à l'informatique, on ne trouve aucun questionnement relatif à la possible influence de l'ordinateur sur la perception du statut du texte biblique. Aland, comme la plupart des intellectuels de son temps, comprend qu'il n'est « rien de plus qu'un outil. Il ne sait faire que ce qu'on lui donne comme tâche à accomplir. [...] L'introduction de l'ordinateur ne signifie absolument pas la mécanisation de la critique textuelle du Nouveau Testament » et ne fait que lui venir en aide ${ }^{42}$. On croirait entendre un écho à l'objection de Lovelace popularisée par Turing, l'ordinateur ne peut pas être à l'origine de quelque chose. Le débat mené au 19e siècle par Lovelace, puis dans les années cinquante par Turing, n'a pas toutefois encore rejoint l'ensemble des sciences humaines.

C'est ainsi qu'on voit Bonifatius Fischer reconduire ce débat. Son article illustre de manière claire les réticences que l'ordinateur va inspirer aux

40 Aland, «Novi Testamenti Graeci », p. 174: «Aber der beschriebene Aufbau der Editio maior critica ergibt sich zusätzliche auch noch daraus, dass für den Aufbau dieser Ausgabe wie vor allem für ihre Auswertung der Computer herangezogen wird. Nur die beschriebene Form macht den Einsatz des Computers möglich ».

41 Aland, « Novi Testamenti Graeci », p. 174 : «Der Computer hat seine eigenen Gesetze, allein die Bezeichnung der Varianten mit Ziffern und Buchstaben ermöglicht es ihm, den kritischen Apparat zu lesen und zu verarbeiten. Seine Benutzung bedeutet eine entscheidende Hilfe bereits beim Aufbau des kritischen Apparates. Wir brauchen damit nicht zu warten, bis etwa die letzte Kollation der letzten griechischen Handschrift und auch die Auswertung des letzten Kirchenvaters vollständig vorliegt, um den kritischen Apparat dann stückweise zusammenzusetzen und innerhalb jeder Variante mühsam die richtige Reihenfolge Papyri, Majuskeln, Minuskeln, Lektionare, usw. herzustellen, wobei dann jedes Mal noch die genaue numerische Reihenfolge beachtet werden muss. Jeder dieser Arbeitsgänge bietet, wie der Erfahrene weiss, unzählige Ansätze zu Fehlern. Bei der Arbeit mit dem Computer kann das Material abschnittsweise eingegeben werden, wenn man will jede Handschrift nach Abschluss der Kollation ; in Minutenschnelle bringt der Computer das Material in die vorgeschriebene Reihenfolge, bzw. ordnet er alle Lesarten der Handschrift an allen Stellen richtig ein ».

Aland, « Novi Testamenti Graeci », p. 175-176 : «Der Computer ist nicht mehr als ein Werkzeug. Er kann nur tun, was man ihm zu tun aufgibt. [...] Der Einsatz des Computers bedeutet keine Mechaniesierung der neutestamentlichen Textkritik, er stellt ihr nur Hilfsmittel für ihre Arbeit zur Verfügung, die sie vereinfachen und ihr eine bisher nicht mögliche Intensität und Extensität geben, vorausgesetzt, dass sie richtig genutzt werden ». 
chercheurs-mêmes qu'il va fasciner. A plus d'un siècle de distance, Bonifatius Fischer adopte d'entrée - mais sans être conscient de ces références - la position de Menabrea et Lovelace, en affirmant que l'ordinateur ne pourrait rien comprendre de lui-même, ni être à l'origine de quelque chose, dans la même veine qu'Aland. Il ne laisse en revanche pas un soupçon d'ouverture à des progrès futurs, au contraire de Lovelace :

Nous devons être clairs quant au fait que l'ordinateur, par principe, peut seulement reconnaître des signes et leur alignement, les comparer les uns avec les autres, et réaliser diverses opérations afférentes, d'une manière externe : il n'a aucune compréhension de la signification ou du contexte des mots ou phrases singuliers. De manière similaire, les machines à traduire, dont nous avons entendu parler et à propos desquelles nous avons lu, n'ont aucune compréhension du langage. ${ }^{43}$

De fait, Fischer se montre globalement peu enthousiaste pour l'usage de l'ordinateur, estimant qu'il ne convient pas d'y archiver à grands frais « ce qu'on peut facilement trouver dans les livres existants $»^{44}$. Enfin, aux deux tiers de l'article et « après tant de pessimisme », comme il le commente lui-même, il concède qu'il est un domaine où l'ordinateur est « de très grande importance pour l'étudiant du Nouveau Testament, dans lequel il ouvre en effet une nouvelle dimension et rend possible ce dont le chercheur n'aurait pas même osé rêver jusque-là : celui de la critique textuelle ${ }^{45}$. Avec cette remarque est mise en place la dichotomie des usages de l'informatique entre critique textuelle et théologie, une dichotomie qui continue à se refléter dans les travaux actuels, comme le montrent les points 1.2 (critique textuelle) et 1.4 (théologie). Fischer poursuit en présentant avec enthousiasme le travail informatique prévu « sur le continent », pour l'Epître de Jacques dans le cadre de l'ECM ${ }^{46}$, mais non sans

43 Fischer, «The use of computer », p. 299-300 : « We must be clear then in principle that the computer can only recognize signs and their succession, compare them with one another, and perform various related operations, in an external way : it has no understanding of the meaning or context either of individuals words or of sentences. Likewise the translation machines of which we have heard and read have no understanding of language ». Voir aussi p. 304 : « the machine has no understanding of the content ».

44 Fischer, «The use of computer», p. 303.

45 Fischer, « The use of computer », p. 304 : «After so much pessimism we come at last to a field where the computer is of great importance to the student of the New Testament, indeed where it opens up a new dimension and makes possible what hitertho the scholar had not even dared to dream of : that is, in textual criticism ».

46 Fischer, « The use of computer», p. 307-308. 
conclure en répétant encore une fois son credo en la supériorité des capacités humaines inégalables par la machine ${ }^{47}$.

Il me paraît très important de garder en tête ce positionnement de départ. En effet, si dans le discours des chercheurs en sciences bibliques contemporains l'utilité et l'efficacité de l'ordinateur paraît désormais complètement intégrée $^{48}$, la répartition claire entre homme et machine, présente dès les travaux d'Aland et Fischer, n'a pas été remise en question, de même que le statut du texte biblique. Et pourtant, Liv Ingeborg Lied résume en quelques phrases claires la transformation en cours du champ des études bibliques dans son ensemble par la digitalisation des manuscrits :

Plusieurs sous-champs du vaste ensemble des études bibliques ont été caractérisés par la division du travail entre éditeurs et exégètes, laquelle a produit à son tour une division entre chercheurs qui sont entraînés à travailler sur les manuscrits et ceux qui ne le sont pas. Avec l'augmentation de la mise à disposition visuelle, les chercheurs qui sont exégètes pourraient avoir à amener de nouveaux outils interprétatifs dans l'étude des manuscrits et de leurs variantes textuelles, en plus de ceux de leurs collègues qui se s'y sont traditionnellement intéressés, et ils pourraient ainsi provoquer l'émergence de nouvelles approches. Pendant les dernières décades, nous avons assisté à lémergence d'un intérêt général pour la matérialité des artefacts, le rôle des médias, aussi bien que pour l'esthétique, le scribalisme et les cultures scribales, les paratextes et marginalia, ainsi que les pratiques des lecteurs dans ces champs. Les manuscrits digitalisés sont devenus une source de matériel disponible pour des chercheurs influencés eux-mêmes par ces récents débats dans la recherche. ${ }^{49}$

$47 \quad$ Fischer, « The use of computer », p. 308.

48 Voir notamment Houghton, « Electronic Transcriptions ».

49 L. I. Lied, « Digitization and Manuscripts as Visual Objects: Reflections from a Media Studies Perspective », dans D. Hamidović - C. Clivaz - S. Bowen Savant (éd.), Ancient Manuscripts in Digital Culture (DBS 3), Brill, 2019, p. 15-29 ; ici p. 24 : « Many subfields under the larger umbrella of biblical studies have been characterized by a division of labor between editors and exegetes, which has produced a division between scholars who are trained in working on manuscripts and scholars who are not. With the increased visual availability, scholars who have been exegetes may bring new interpretative tools to the study of manuscripts and their various texts, beyond those their colleagues have traditionally taken interest in, and hence giving rise to new approaches. During the last few decades, we have seen a general surge of interest in the materiality of artifacts, in the role of the medium, as well as in aesthetics, scribalism and scribal cultures, paratexts and marginalia, and reader practices in these fields. Digitized manuscripts have become an available source material for scholars who are influenced by these recent research debates». 
L'ampleur du tournant est également décrite par Hugh Houghton comme un « changement fondamental dans la création de l'édition » digitale du Nouveau Testament, qui se trouve désormais avoir une double tâche : « éditer les documents individuels et créer un archétype électronique pour chaque témoin du livre biblique concerné. Ensuite seulement utiliser cette information pour éditer le texte lui-même ${ }^{50}$. On trouve dans cet article fondamental de Houghton un résumé clair de l'évolution des transcriptions électroniques, que je donne ici en note ${ }^{51}$. Il permet aussi à ses lecteurs d'acquérir une vision synthétique des transformations de l'apparat critique :

L'apparat critique est compilé automatiquement à partir de divers fichiers, se servant d'un algorithme qui améliore l'alignement, et crée des méta-fichiers pour aider à la normalisation des données. Ce processus a au moins quatre avantages par rapport à la méthode précédente: l'accomplissement de la tâche mécanique de compilation par un ordinateur est beaucoup plus rapide, moins sujette à l'erreur humaine, reproductible et reconfigurable. Une collation peut être reconduite pour les mêmes fichiers avec des paramètres différents ou pour une sélection de témoins différents. ${ }^{52}$

$50 \quad$ Houghton, «Electronic Transcriptions », p. 134 : to « edit the individual documents, creating an electronic archetype of each witness for the required biblical book. Only then can they proceed to use this information to edit the text itself ».

Houghton, « Electronic Transcriptions », p.136: « The first generation of electronic transcriptions, created for use with the original Collate software, were plain-text files with basic tags for markup, produced in a standard text editor. These were converted in a separate process to a more advanced format for publication (first SGML, then XML). The Workspace for Collaborative Editing project produced the browser-based Online Transcription Editor in 2013. This enabled transcribers to work directly on XML files in a display, which matched the published transcriptions, the markup being hidden behind the scenes. Not only was the aim to standardise the markup and deliver formally correct files, but this procedure also meant that transcriptions could be published online and distributed immediately. One of the strengths of XML encoding corresponding to the TEI Guidelines is that each file is complete in itself, with a standard form of markup, which is not only largely readable by humans but also actionable by machines. This is vital for the long-term sustainability of these files as well as their availability for re-use, as discussed below. The Online Transcription Editor supports a wide variety of TEI-compatible features which can be added as enhancements to standard transcriptions, such as formatting, annotations and other paratextual features ». 
Comme on le voit, le processus éditorial décrit par Houghton reste dans le droit fil de l'édition critique basée sur les stemma. Il s'agit ici d'une simple observation, mais qui explique pourquoi on ne trouve pas non plus chez Houghton de réflexion sur le statut du texte biblique dans lédition et la culture digitale. Un tel questionnement n'est pas non plus présent dans la monographie de Wasserman et Gurry, qui part du donné des vingt-sept livres du Nouveau Testament comme corpus absolument stable, un texte destiné à être lu et prêché : le public des chrétiens pratiquants fait ici partie intégrante du projet de recherche ${ }^{53}$. Cet ouvrage introductif est absolument fascinant à lire, d'abord parce qu'il présente avec une grande clarté la Coherence-Based Genealogical Method (свGM $)^{54}$ et permet un débat fructueux. Ensuite parce qu'il témoigne exactement d'un moment que je qualifierai « d'entre-deux » dans la critique textuelle du Nouveau Testament (NTTC) : l'intégration de l'ordinateur est à l'œuvre dans une structure qui n'a pas encore remis en question ses fondamentaux hérités de la culture imprimée.

Wasserman et Gurry signalent en effet clairement que le changement le «plus significatif et le plus controversé » de la СвGM est l'abandon des grands textes types, en vigueur depuis le 18 e siècle ${ }^{55}$ et rapportent la racine de cette

A collation can be re-run from the same files with different settings or a different selection of witnesses ».

Wasserman - Gurry, A New Approach to Textual Criticism, p. 9 : « Textual criticism is needed for all twenty-seven books of the New Testament because, so far as we know, none of the autographs still exist; all were lost to the ravages of time and use. [...] Which text should be read? Which should be applied? Which should be preached? This is one of the questions that textual criticism tries to answer ».

54 Pour une définition, voir G. Mink, « The Coherence-Based Genealogical Method. What is about?», <https://www.uni-muenster.de/INTF/Genealogical_method.html> : «The Coherence-Based Genealogical Method is based on the following assumptions: In a textual tradition where all the copies have survived and where the source, or (in case of contamination) the sources, are also known, as well as the origin of every reading in every copy, the genealogical interrelationships between all the variants at any place of variation must appear in a global stemma of the witnesses as genealogical relationship between coherent fields of relationships between witnesses ».

55 Wasserman - Gurry, A New Approach to Textual Criticism, p. 13: « The свgм has also introduced several far-reaching changes in how the editors view and describe the history of the text. The most significant and, for that reason, controversial is that it has convinced the editors to abandon the concept of text-types traditionally used to group and evaluate manuscripts. For more than two centuries, New Testament scholars have spoken about the "Alexandrian," "Western," "Byzantine" (or "Eastern" or "Syrian"), and sometimes "Caesarean" texts. An early pioneer, J. A. Bengel (1687-1752), took on the task of sorting out the wealth of source materials in order to reconstruct the earliest text of the New Testament. He divided the textual witnesses into groups that he called "nations" and "families." J. S. Semler (1725-1791) and J. J. Griesbach $\left(1745^{-1812}\right)$ refined Bengel's scheme by 
transformation précisément à l'introduction de l'informatique dans la NTTC. Ils restent prudents quant à la réception en cours de cette transformation ${ }^{56}$ : « parce que l'ordinateur peut garder la trace de tous ces témoins et de leur place dans la transmission, il n'y a pas besoin de les grouper en quelques textes types et de relier ces groupes ${ }^{57}$. Le constat est on ne peut plus clair, et on a ici la validation de ce qui change en profondeur dans la NTTC au sein de la culture digitale. En même temps, nos deux auteurs conservent une perspective lachmanienne avec une nette distinction « entre les manuscrits en tant qu'artefacts physiques et les textes que ces artefacts contiennent », la CBGM ayant « l'intention de relier seulement les textes et non les manuscrits $»^{58}$. On est ici à la croisée entre documents et textes reconstruits, que nous allons retrouver au point suivant dans le parcours interdisciplinaire de notre thématique.

Comme le commente Garrick Allen à propos de l'ECM, en ouverture d'un collectif qu'il vient de publier, The Moving Text ${ }^{59}$, « dans la troisième phase du projet (2017-2020), le matériel sera soumis à analyse en utilisant la CoherenceBased Genealogical Method (СвGM), une méthode stemmatique pour évaluer et faire des scénarios de génétique textuelle de la tradition, dans un effort pour créer le texte le plus ancien possible, pour chaque unité de variante ${ }^{60}$. Ce collectif demande à être observé dans le cadre de notre problématique. En effet, il s'annonce comme « interdisciplinaire » dans son sous-titre, mais c'est une interdisciplinarité à entendre à l'intérieur des études textuelles bibliques. Nous retrouvons ici l'absence de réel dialogue interdisciplinaire, au sens large du terme, sur la perception des éditions digitales de la part des chercheurs en

connecting these textual groupings to geographical areas where the text might have been revised (apart from the normal copying) ».

56 Wasserman - Gurry, A New Approach to Textual Criticism, p. 16 : «The rejection of the concept of text-types as a means of understanding the history of the text is significant. What fruit this will bear in the long term remains to be seen. It also remains to be seen whether New Testament scholars more generally will accept the свGм as a viable replacement to text-types ».

57 Wasserman - Gurry, A New Approach to Textual Criticism, p. 14 : «Because the computer can keep track of all these witnesses and their place in the transmission, there is no need to group them into a few text-types and relate these groups ».

$5^{8}$ Wasserman - Gurry, A New Approach to Textual Criticism, p. 10.

59 G. V. Allen - C. R. Brewer - D. F. Kinlaw III (éd.), The Moving Text. Interdisciplinary Perspectives on David Brown and the Bible, SCM Press, 2018.

60 G. V. Allen, « Text and Tradition : David Brown and New Testament Textual Criticism», dans Allen - Brewer - Kinlaw III (éd.), The Moving Text, p. 3-16 ; ici p. 5 : «In the third phase of the project (2017-20) the material will be submitted to analysis using the Coherence-Based Genealogical Method (св GM), a stemmatic method for evaluating and plotting the textual genetics of the tradition in an effort to create the oldest attainable text in each variation unit». 
Nouveau Testament. On ne trouve pas non plus dans l'article introductif d'Allen de mention des nouvelles éditions grecques du Nouveau Testament ${ }^{61}$, et comme nous l'avons vu, il réaffirme le principe de la quête du texte original comme but de l'ECM.

Par contre, comme son titre principal l'indique, ce collectif prend au sérieux le fait que la NTTC souhaite désormais non seulement établir le texte original le plus ancien possible, mais est également très intéressée aux variantes et développements de la tradition chrétienne ${ }^{62}$. Une telle perspective est aussi celle du projet La Bible en ses traditions pour le texte biblique au sens large : « Plutôt qu'un texte unique comme les Bibles ordinaires, La Bible en ses Traditions entend refléter la diversité des traditions textuelles. [...] Nous ne visons pas le même but que la critique textuelle classique - établir la forme la plus pure, la plus primitive du texte grec $»^{63}$.

Allen commence, quant à lui, par rendre hommage aux portes ouvertes par l'ouvrage novateur de David Parker, The Living Text of the Gospels, qui soulignait « l'interjeu continu entre l'Ecriture - le texte copié - la tradition - la personne engagée par et dans l'Eglise pour le processus de copie ${ }^{64}$. Mais le but du collectif est de croiser NTTC et les travaux que David Brown a développés dans une monographie en 1999 quant à l'idée que les traditions sont à l'œuvre dans l'énoncé et l'interprétation du texte canonique ${ }^{65}$. Allen conclut à la compatibilité de l'approche de Brown avec l'Ecm, l'appart critique étant une « carte d'orientation » des traditions chrétiennes pour le lecteur ${ }^{66}$.

S'il y a donc le désir théorique, dirais-je, dans l'approche d'Allen d'honorer le moving text du corpus biblique, via le double héritage de Parker et Brown, manquent ici les voix interdisciplinaires pour manifester les transformations du statut du texte et de la quête de l'original provoquées par la culture digitale. La ligne de fond d'Allen reste celle de la culture textuelle imprimée :il pense les

61 Voir note 28 ci-dessus et chapitre 1, p. 31-32.

62 Allen, «Text and Tradition », p. $3:$ « In fact, textual scholars are more and more interested in what textual variation and the shape of textual culture tell us about tradents (i.e. the people who produced manuscripts), and the development of Christian tradition ».

63 Ecole Biblique et Archéologique Française de Jérusalem (éd.), La Bible en ses traditions. Définitions suivies de Douze Etudes, Jérusalem, 2011, p. 21.

64 D. C. Parker, The Living Text of the Gospels, Cambridge Universit Press, 1997, p. 15 ; cité par Allen, « Text and Tradition », p. 7 : the « continuing interplay between Scripture - the text copied - and the tradition - the person engaged in the process of copying in and for the church ».

65 D. Brown, Tradition and Interpretation: Revelation and Change, Oxford University Press, 1999, p. 31 et 208-209 ; voir Allen, « Text and Tradition », notamment p. 8.

66 Allen, « Text and Tradition », p. 13. 
traditions en termes de textes et de documents ${ }^{67}$; ce lien demeurera de fait tant que l'ECM continue à viser une double édition papier et électronique ${ }^{68}$.

A ce stade, on perçoit bien les traits récurrents - chez les auteurs évoqués - qui ont retardé le débat, au sein des sciences bibliques et du Nouveau Testament en particulier, sur le statut du texte dans la culture digitale : une quasi absence de débats avec des DHers sur les éditions digitales et donc l'absence d'une discussion sur la quête du texte original; l'attachement conservé à la С ВGM et donc à la quête du texte original, sans réflexion sur la signification de l'émergence de nouvelles éditions critiques du NT. Cet état de fait, dont nous avons les racines chez Aland et Fischer, permet de comprendre pourquoi une monographie sur la Bible dans la culture digitale s'est fait attendre soixante ans : l'absence de prise de conscience, chez les tenants de la NTTC, de la transformation du statut du texte dans la culture digitale. Mais comme nous le verrons au point 1.4, les plaques tectoniques des continents de la recherche ne se sont pas encore rejointes : en effet, Jeffrey Siker ne discute pas, dans sa monographie Liquid Scripture, les transformations en cours des éditions critiques du Nouveau Testament grec ${ }^{69}$. La division entre éditeurs et exégètes, soulignée par Liv Ingeborg Lied, est toujours là70 ${ }^{70}$ Prenant acte de cette division en théologie, le point 1.3 se propose de scruter l'état des lieux dans une perspective interdisciplinaire.

\subsection{Regards interdisciplinaires sur le statut du texte digital}

C'est dès octobre 2009 que j'ai défendu pour ma part l'idée qu'il fallait se questionner sur le statut du texte biblique dans la culture digitale, lors de ma première conférence intégrant ce sujet, en partant de remarques perspicaces de Roger Chartier et Umberto $\mathrm{Eco}^{71}$. Roger Chartier relève la très grande flexibilité, voir l'impermanence du texte numérique :

Le monde du texte numérique est un monde où les textes sont déployés, repris, réécrits, où une écriture s'écrit dans une écriture déjà là, un monde où le lecteur intervient non pas sur les marges du texte, mais dans les textes eux-mêmes, un monde où comme l'avait rêvé parfois Foucault,

67 Allen, « Text and Tradition», p. $6:$ « Can the documents that transmit the NT, with their varying presentations of their texts and numerous variants, function as points (sources?) of revelation?». Allen, «Text and Tradition», p. $6:$ « the edition will be published in both paper and electronic forms ».

69 Siker, Liquid Scripture.

70 Voir citation p. 175 ci-dessus.

71 Clivaz, « The New Testament at the Time of the Egyptian Papyri », part. p. 18-25. 
s'effacerait l'assignation au nom propre, où s'effacerait la « fonction auteur » dans une sorte de textualité formée de nappes de discours toujours repris et liés à l'échange permanent entre producteurs et lecteurs - mais des lecteurs à leur tour auteurs ${ }^{72}$.

Umberto Eco va plus loin encore en affirmant carrément que la notion de texte original « certainement disparaît » dans la culture digitale, où le problème est constitué par

les altérations que je peux faire, moi, sur les textes des autres. Supposons que je décharge sur mon ordinateur La Critique de la raison pure, que je commence à l'étudier, et que j'écrive tous mes commentaires entre les lignes, ou bien je suis doué d'un fort esprit philologique et je peux reconnaître mes commentaires, ou bien, trois années plus tard, je ne saurai plus ce qui est de moi et ce qui est de Kant. Nous serions comme ces copistes du Moyen Âge qui corrigeaient automatiquement le texte qu'ils copiaient parce que cela leur semblait normal, d'où le risque que l'esprit philologique s'en aille en eau de boudin. Mais, là aussi, le risque pour le jeune étudiant est qu'il ne s'aperçoive plus qu'il a manipulé le texte. Les milieux scientifiques et universitaires resteraient les garants de cette vigilance philologique ${ }^{73}$.

On ne saurait mieux illustrer le choc frontal qui atteint la philologie. En même temps, si les propos de Chartier et Eco sont on ne peut plus clairs, ils ont une tonalité prédictive qui laissent les lecteurs dans le questionnement : comment, dans le réel du travail minutieux des éditeurs critiques, un tel choc se fait-il ressentir ou non? De fait, lire les propositions qui fusent de part et d'autre dans l'étude des éditions critiques digitales depuis quelques années est une aventure passionnante, car on y voit un nouveau monde en fusion, charriant de gros blocs du passé utiles à l'aventure, mais avec aussi un pouvoir innovant assez impressionnant.

Pour en rester à la philologie pointée par Eco, je soulignerai l'article de 2010 d'Ernst Thoutenhoofd qui part de cet « esprit philologique fort » exprimé par

72 R. Chartier, Les métamorphoses du livre: Les rendez-vous de l'édition. Le livre et le numérique, Bibliothèque du Centre Pompidou, 2001, p. 16-17.

73 U. Eco - G. Origgi, « Auteurs et autorité : un entretien avec Umberto Eco », dans G. Origgi - N. Arikha (éd.), Texte-e : Le texte à l'heure de l'Internet, Bibliothèque Publique d'Information, 2003, p. 215-230 ; ici p. 227. 
Eco, ou de l'idée de sens ou présence originels à reconstruire ${ }^{74}$. Constatant, comme Eco, qu'on quitte « la référence insistante à l'idéal d'un texte ou d'une 'source' originaux », il propose de joindre l'analyse des sciences sociales à celle des sciences humaines pour refonder la philologie :

La transformation intellectuelle de la très ancienne attention analytique aux origines textuelles des idées, dans les conditions de production digitale, fait de l' « e-philologie » un cas d'école très pertinent pour l'analyse des sciences sociales, comme cette dernière est concernée de manière exponentielle par le développement hétérogène d'idées circulant parmi les acteurs (humains et machines), et réparties dans l'espace et le temps par le moyen de techniques et technologies numériques. Il semble à présent clair que les techniques digitales avancées, telles qu'utilisées dans les corpus linguistiques et l'e-philologie, offrent de nouvelles approches pour capturer la nature hétérogène des traductions du savoir via les dimensions de l'espace, du temps et du but ${ }^{75}$.

Quelques années plus tard, sans avoir besoin de faire appel aux sciences sociales en tant que telles, Elena Pierazzo démontre dans sa monographie que l'e-philologie s'inscrit bel et bien dans les interactions sociales, et que chercheurs, producteurs et utilisateurs, sont l'un des facteurs principaux de transformation de l'édition critique :

C'est un état de fait que la véritable distinction entre le travail éditorial et le produit éditorial a maintenant été remise en question, parce que les outils utilisés par les éditeurs dans leur travail éditorial sont eux-mêmes rendus accessibles aux utilisateurs : c'est ce que Gregory Crane définit comme l' «e-philologie », dans laquelle la fonction éditoriale est parta-

74 E. D. Thoutenhoofd, « Presence beyond digital philology », dans W. van Peursen - E. D. Toutenhoofd - A. van der Weel (éd.), Text Comparison and Digital Creativity. The Production of Presence and Meaning in Digital Text Scholarship, Brill, 2010, p. 269-289.

Thoutenhoofd, « Presence beyond digital philology », p. 285 : « The intellectual transformation of this longstanding analytical attention to the textual origins of ideas under digital conditions of production makes 'e-philology' a highly relevant casus for social science analysis, as the latter is increasingly concerned with the heterogeneous development of ideas streaming among actors (humans and machines), and distributing in space and time by way of digital techniques and technologies. It now seems clear that advanced digital techniques, such as used in corpus linguistics and e-philology, offer new approaches for capturing the heterogeneous nature of knowledge-translations across dimensions of place, time and purpose ». 
gée par les éditeurs et les utilisateurs, et dans laquelle le texte est décomposé, analysé et produit par des algorithmes ${ }^{76}$.

A l'extrême ouverture de ce travail interactif se tient le crowdsourcing editing, en test, et dont Pierazzo offre une première évaluation dans son ouvrage ${ }^{77}$. On est bien au-delà des propos d'Umberto Eco qui voyait encore une certaine philologie protégée par « les milieux scientifiques et universitaires ». Mais diverses modalités d'édition collaborative existent et sont d'ores et déjà efficaces, à commencer par la New Testament Virtual Room of Manuscripts (NTVRM), déjà signalée $^{78}$, et que Pierazzo ne mentionne pas.

Un tel contact interdisciplinaire - cette fois-ci des autres branches des sciences humaines vers la théologie - serait pourtant intéressant, car le milieu de la recherche sur les manuscrits du Nouveau Testament représente un cas d'école particulier, en raison du nombre de manuscrits, de leur variété dans le temps, et de l'implication forte des chercheurs de ce milieu, comme je l'avais décrit dans un article de $2013^{79}$. Ce qui est en place dans ce lieu interactif est un modèle particulier et novateur. C'est actuellement une question ouverte de savoir jusqu'à quel point la NTVRM servira de modèle alternatif à la publication à venir de la 2ge édition du Nestle-Aland. C'est ici que se joue exactement le floutage de la frontière entre éditeurs et users des éditions : ceux qui sont en train de transcrire les manuscrits néotestamentaires dans la NTVRM sont déjà en train de poser les briques de l'édition.

Un stade supplémentaire est celui franchi par l'édition en ligne d'Homère, Homer Multitext ${ }^{80}$, qui fait le choix de valoriser les manuscrits en version diplomatique, plutôt que d'en faire l'édition critique ${ }^{81}$. Ce projet manifeste le grand tournant de la culture digitale éditoriale décrite par Wido van Peursen comme celui qui mène des textes aux documents ${ }^{82}$. La valorisation d'un manuscrit pour lui-même, maintenant qu'il est si facile d'y accéder en ligne, est

76 Pierazzo, Digital Scholarly Editing, p. 16.

77 Pierazzo, Digital Scholarly Editing, p. 22 : «Crowdsourcing is an inclusive label that contains a wide range of phenomena and types of activity; an exhaustive classification of these phenomena in an academic context has been attempted only recently (Dunn and Hedges 2012), but an analysis of its viability, relevance and significance within an editorial context has not yet been considered in full. Without any pretension of being exhaustive, I propose here such an evaluation ».

78 INTF \& ITSEe, New Testament Virtual Manuscript Room, <http://ntvmr.uni-muenster. de/> ; voir chapitre 1, p. 33 .

79 C. Clivaz, «Internet Networks and Academic Research ».

$80 \quad$ Homer Multitext, <http://www.homermultitext.org/>.

$81 \quad$ Voir Clivaz, « Homer and the New Testament», p. 3-5.

82 Peursen, « Text Comparison and Digital Creativity »; signalé dans le chapitre 1, p. 17. 
certainement appelée à se développer dans tous les domaines des sciences humaines. Du côté du Nouveau Testament, c'est bien sûr le projet phare du Codex Sinaiticus qui illustre le mieux ce tournant ${ }^{83}$ : le donné biblique est ici celui d'un manuscrit exactement. C'est aussi le choix que nous avons fait en équipe pour un projet du Fonds National Suisse sur le seul manuscrit trilingue grec-latin-arabe connu pour le Nouveau Testament, le Marciana Gr. Z. 11 $(379)^{84}$.

Lattention soutenue, dans divers domaines des sciences humaines, à la valorisation et à l'étude digitales du document comme activité supplémentaire à celle de l'édition critique, entraînera sans doute de profondes transformations dans la science de l'ecdotique, l'étude et l'édition des manuscrits. Nous n'avons pas à cette heure de monographie qui ait pris cette problématique à bras-lecorps : le temps est d'abord à l'exploration pour certains, alors que d'autres revisitent les fondamentaux de la branche en tentant d'évaluer l'impact de la culture digitale. C'est le cas notamment de l'article d'Odd Einar Haugen et Daniel Apollon, «The Digital Turn in Textual Scholarship » ${ }^{85}$. Les auteurs annoncent d'entrée travailler à la croisée des diverses approches répertoriées dans l'histoire de la discipline ${ }^{86}$, en particulier l'approche historique du texte et sa transmission, basée sur les travaux de Karl Lachmann (1793-1851) ${ }^{87}$. Dans ce type d'approche historique, «plutôt que détrôner l'éditeur, la critique textuelle menée de manière précoce à l'aide d'un ordinateur a confirmé la position centrale de celui-ci ou celle-ci, ajoutant des outils et élargissant son espace décisionnel ${ }^{88}$. C'est bien le type de constat qu'on lit aussi chez Wasserman et Gurry, où les décisions principales restent clairement rapportées à l'éditeur et non à la machine ${ }^{89}$.

83 Codex Sinaiticus, <http://codexsinaiticus.org/>.

84 HumaReC, $<$ https://humarec.org/>.

85 O. E. Haugen - D. Apollon, « The Digital Turn in Textual Scholarship. Historical and Typological Perspectives », dans Digital Critical Editions (Topics in the Digital Humanities), D. Apollon - C. Bélisle - P. Régnier (éd.), University of Illinois Press, 2014, édition Kindle.

86 Haugen - Apollon, « The Digital Turn », édition Kindle, 1. 787: « The approach defended in this chapter reflects the belief that the self-contained nature of texts, as advocated by this New Criticism (looking inward), and the awareness of the organic relationship of texts to their world (looking outward) can benefit from a historical approach to the text and its transmission (looking backward). The purpose of this chapter is to show how these three perspectives may shape digital text scholarship ».

87 Haugen - Apollon, « The Digital Turn », édition Kindle, 1. 801.

88 Haugen - Apollon, «The Digital Turn », édition Kindle, 1. 887 : « Rather than dethroning the editor, early computer-assisted textual criticism has confirmed his or her central position, adding tools and enlarging his or her decision space».

89 Wasserman - Gurry, A New Approach to Textual Criticism, p. 11. 
Dans cette rubrique, Haugen et Apollon font tout naturellement place à la recherche sur les manuscrits du Nouveau Testament, dès le milieu des années soixante, et dès 1979 existe une liste variée de diverses méthodologies existantes au sein de la reconstruction historique des textes dans l'édition digitale, dont « des méthodes orientées vers les graphes, telle l'analyse phylogénétique, un pilier méthodologique de l'évolution génétique. [...] Peter Robinson et des collègues ont soutenu l'idée que l'analyse phylogénétique pouvait offrir un long parcours pour résoudre le problème inhérent à toute recension $»^{90}$.

Comme on l'a vu, Peter Robinson est l'un des chercheurs en sciences humaines qui a le plus collaboré avec des collègues en Nouveau Testament sur ces questions d'édition digitale. Du côté des néotestamentaires, David Pastorelli utilise le software PHYLIP (Phylogeny Inference Package), créé par Joseph Felsenstein, à l'Université de Washington et en libre accès ${ }^{91}$. PHYLIP fournit des algorithmes aux phylogéniticiens et biologistes, et Pastorelli s'en est notamment servi pour une analyse quantitative de Mc 6 et $9^{92}$. Il travaille notamment en collaboration avec Caroline Macé et l'équipe de phylogénéticiens de l'Université catholique de Louvain-la-Neuve, spécialistes de littérature patristique $^{93}$. Une telle analyse a également été conduite, par exemple, dans le champ de la littérature halachique ${ }^{94}$.

La méthode ne semble toutefois pas tenir ses promesses, tant dans l'évaluation d'Haugen et Apollon ${ }^{95}$ que de Pierazzo qui souligne que «cette métho-

90 Haugen - Apollon, «The Digital Turn », édition Kindle, 1. 859 : «graph-oriented methods, such as phylogenetic analysis, a methodological pillar of evolutionary genetics. [...] Peter Robinson and colleagues have argued that phylogenetic analysis can come a long way toward solving the chronological problem inherent in any recension ».

$91 \quad$ PHYLIP, <http://evolution.genetics.washington.edu/phylip.html>.

92 Pastorelli, « The Chester Beatty I Papyrus (P45) », p. 298-299.

93 C. Macé - T. Schmidt - J.-F. Weiler, « Le classement des manuscrits par la statistique et la phylogénétique : le cas de Grégoire de Nazianze et de Basile le Minime », Revue d'Histoire des Textes 31 (2001), p. 243-273 ; C. Macé - P.V. Baret - A.-C. Lantin, Philologie et phylogénétique: regards croisés en vue d'une édition critique d'une homélie de Grégoire de Nazianze, dans Digital Technology and Philological Disciplines (Linguistica Computazionale 20-21), A. Bozzi - L. Cignoni - J.-L. Lebrave (éds.), Istituti Editoriali e Poligrafici Internazionali, 2004, p. 305-341 ; C. Macé - P.V. Baret, « Why Phylogenetic Methods Work: The Theory of Evolution and Textual Criticism », dans The Evolution of Texts : Confronting Stemmatological and Genetical Methods. Proceedings of the International Workshop held in Louvain-laNeuve on September 1-2, 2004 (Linguistica Computazionale 24), C. Macé - P.V. Baret A. Bozzi (éd.), Istituti Editoriali e Poligrafici Internazionali, 2006, p. 89-108.

94 Yorav - Dagan - Graur, « An Exploratory Study ».

95 Haugen - Apollon, « The Digital Turn », édition Kindle, 1. 873 : «Unfortunately, we do not have any complete manuscript filiations from Antiquity or the Middle Ages; we have only fragments of unknown proportions. The 'true' filiation of a text can thus never be ascertained, but only approximated ». 
dologie n'a pas été absente de critique. [...Son] problème principal réside dans la difficulté à déterminer comment pondérer les lectures variantes, et si c'est tout simplement possible ${ }^{96}$. Le cas de l'analyse phylogénétique nous intéresse particulièrement dans notre questionnement sur le statut du texte, car elle reflète d'une part le conservatisme du rôle éditorial, et ouvre d'autre part au questionnement sur la symbolique de la représentation, discutée à la fin de ce point 1.3. Haugen et Apollon commentent ainsi le rôle de l'éditeur digital :

Ces récentes avancées méthodologiques ont le contre-effet apparemment paradoxal de confirmer la position et le rôle de l'éditeur au centre du processus éditorial. Alors que les tenants de base de la méthode généalogique n'ont pas été remis en question depuis l'époque de Lachmann, et que la méthode, idéalement, devrait encore être regardée comme valable, ce n'est pas toujours praticable ${ }^{97}$.

On retrouve dans ces quelques phrases la tension relevée au sein de l'introduction de Wasserman et Gurry : sur ce plan, aucune différence entre critique textuelle du Nouveau Testament et analyse générale de l'édition critique digitale. De fait, Haugen et Apollon se révèlent illustrer la même tension entre héritage et innovation que Wasserman et Gurry, mais à propos de la conception de la page et de l'écriture. En effet, ils indiquent d'une part que le changement principal de l'écriture en ligne est la « résilience d'une vision de l'espace à deux dimensions » ${ }^{98}$, mais réaffirment d'autre part la nécessité de la page en raison de la «nature de l'écriture elle-même», « bidimensionnelle, linéaire et séquentielle $»$, bref « en tous les cas, l'écriture demeure linéaire ${ }^{99}$.

On est ici à l'opposé de ce que le chapitre 3 de ce livre a cherché à démontrer à propos de l'écriture digitale, de son lien au code, à la multimodalité et à la

96 Pierazzo, Digital Scholarly Editing, p. 17 : « This methodology has not been immune from criticism. [...] The main problem with this approach is represented by the difficulty of determining how, and it at all, to weigh variant readings ».

97 Haugen - Apollon, «The Digital Turn », édition Kindle, l. 873: « These recent metholological advances have the seemingly paradoxical side effect of confirming the position and role of editor at the center of the editing process. While the basic tenets of the genealogical method have not been questioned since the time of Lachmann, and the method, ideally should still be regarded as valid, it is not always praticable ».

98 Haugen - Apollon, « The Digital Turn », édition Kindle, l. 1043.

99 Haugen - Apollon, « The Digital Turn », édition Kindle, l. 1057 : « the nature of writing itself [:] writing - or script - is inherently two-dimensions, linear, and sequential. [...] In all cases writing remains linear : it has a starting point and an end point (however, reading may become highly nonlinear) ». 
corporéité en particulier ${ }^{100}$ : Haugen et Apollon n'ont pas fait le passage de l'écriture imprimée à l'écriture digitale dans leur description. Et pourtant, dans l'introduction du collectif que leur article inaugure - une introduction signée par Apollon, Bélisle et Régnier -, il est clairement dit que l'écriture multimodale rend la linéarité insuffisante ${ }^{101}$. Au-delà de cette déclaration liminaire, l'ouvrage ne traite toutefois pas du thème de l'écriture multimodale, à trois brèves mentions près ${ }^{102}$, pas plus que du thème de l'impact de l'oralité dans l'écriture digitale, dont nous avons vu également l'importance au chapitre $3 \mathrm{du}$ présent ouvrage ${ }^{103}$.

D'autres auteurs spécialistes des manuscrits perçoivent bien sûr l'émergence de ces dimensions, par exemple Carrie Schroeder à propos de la multimodalité104. Majoritairement, on retrouve toutefois tant chez les critiques textuels du Nouveau Testament que chez les spécialistes d'éditions digitales la tension entre allégeance et distanciation à Lachmann, l'affirmation de la linéarité bidimensionnelle de l'écriture par Haugen et Apollon faisant figure de clou du spectacle à cet égard. Revisiter cet héritage de l'ecdotique va être le travail de toute une génération. Mais il ne se fera sans doute pas sans la prise de conscience de l'importance des représentations symboliques dans cette discussion, un pas franchi dans un article novateur et encore trop peu reconnu de Charlotte Touati.

Sur le site d'un projet de recherche soutenu par le Fonds National Suisse et Infoclio ${ }^{105}$ et portant sur un manuscrit éthiopien falasha ${ }^{106}$, Charlotte Touati a mis en ligne un article encore non publié ailleurs ${ }^{107}$, qui se questionne sur le bien-fondé de publier un seul manuscrit et revisite des points-clés de l'histoire de l'édition des manuscrits. Il mériterait sans doute quelques approfondissements, mais il contient des idées fondamentales pour ce point 1.3. La thèse de Touati est la suivante :

\footnotetext{
$100 \quad$ Voir chapitre 3, point 4.3.

101 D. Apollon - C. Bélisle - P. Régnier, «Introduction. As Texts Become Digital », dans Digital Critical Editions (Topics in the Digital Humanities), D. Apollon - C. Bélisle - P. Régnier (éd.), University of Illinois Press, 2014, édition Kindle, 1. 644.

102 Apollon - Bélisle - Régnier (éd.), Digital Critical Editions, édition Kindle, 1. 2594, 2837 et 3280 .

103 Voir chapitre 3, notamment point 2.1.

104 Schroeder, « The Digital Humanities as Cultural Capital », p. 43.

105 Infloclio est un service de l'Académie suisse des sciences humaines et sociales : <http:// www.infoclio.ch>.

106 A la découverte des Falashas, $<$ http://falashas.epfl.ch $>$.

107 C. Touati, « Pourquoi éditer un manuscript unique ? Lédition critique des écrits apocryphes : de l'arbre au mycélium », p. 1-13, <https://falashas.epfl.ch/data/sources/textes/Ma nuscrit.pdf>.
} 
Une édition numérique n'est pas une édition assistée par ordinateur. Elle accompagne un changement de conception du texte et de ses réalisations. [...] L'édition critique, assistée ou non par ordinateur, est une utopie mécaniste, qui ne laisse aucun espace entre les copies. Et pourtant c'est une évidence physique : les manuscrits ne naissent pas des manuscrits ${ }^{108}$.

Dans cette optique, elle opère une intéressante relecture critique des travaux d'Auguste Schleier au 1ge siècle, notant de ses emprunts à la biologie et au darwinisme de son temps ${ }^{109}$, non sans y ajouter un purisme de l'origine qui teinte la philologie et l'édition des manuscrits d'un vocabulaire de « contamination ${ }^{110}$. Un tel vocabulaire se retrouve parfois y compris dans l'introduction de Wasserman et Gurry ${ }^{111}$. Osant la mémoire politique et rappelant l'adhésion d'Ernst Hackel, ami et collaborateur de Schleier, à la ligue Pan-germanique, Touati souligne que

toute la démarche de Schleicher est gouvernée par la poursuite de la $U r$ sprache, à savoir l'indo-européen commun ou selon sa terminologie l'indo-germanique ou aryen. Cette quête des origines est une quête de pureté non sans implications idéologiques et politiques ${ }^{112}$.

Elle rappelle aussi qu'en 1995, David Greetham ${ }^{113}$ a mis « en parallèle le développement de la reconstitution stemmatique et une conception monogame et patriarcale de la famille, où seule importe la branche aînée, celle qui transmet le nom par les mâles »114. En abordant cet héritage sous un tel angle analytique, la chercheuse met en évidence l'aspect symbolique d'un paradigme de recherche. Laspect le plus novateur de son article est d'inviter ses lecteurs à

108 Touati, « Pourquoi éditer », p. 11.

109 Touati, «Pourquoi éditer », p. 1: «Philologie, linguistique et biologie ne seraient selon [Auguste Schleicher] que les diverses expressions d'une même science, toutes trois pouvant être modélisées par un stemma (arbre généalogique). De l'analyse des antécédents, il convient de passer à l'examen de la situation présente : alors que les biologistes et les linguistes ont changé de paradigme, remettant en cause des notions qui pourtant semblaient fondamentales dans leur discipline respective, telles que la race, le spécimen, la langue et même le stemma, les philologues peinent à faire évoluer la leur ».

110 Touati, « Pourquoi éditer », p. 6.

111 Wasserman - Gurry, A New Approach to Textual Criticism, p. 21-22.

112 Touati, « Pourquoi éditer », p. 4.

113 D. Greetham, « Phylum-Tree-Rhiozome », Huntington Library Quarterly $5^{8}$ (1995), p. 99126.

Touati, « Pourquoi éditer», p. 6. 
reconsidérer les schémas biologiques sollicités pour décrire notre rapport au texte. L'arbre de la stemmatologie paraît désormais trop linéaire et statique pour rendre compte des développements textuels. C'est déjà l'avis discret de Wasserman et Gurry qui rappellent qu'avant l'arbre, le terme de stemma a le sens de couronne ou guirlande, un usage qui rend mieux compte des derniers développement et représentations de la СвGM ${ }^{115}$.

Touati rappelle quant à elle qu'il y a plus de vingt ans, Greetham souhaitait déjà inviter les philologues à remplacer cette métaphore par celle du rhizome, promue en 1976 par Deleuze et Guattari ${ }^{116}$. Puis elle opte pour le «mycélium », partie souterraine d'un champignon, en s'appuyant sur un article original de Will Derks sur la littérature malaisienne ${ }^{117}$. Derks démontre que

la littérature malaisienne n'a pas besoin d'être écriture pour être prestigieuse et vivante ${ }^{118}$. Bien au contraire, son dynamisme est peut-être proportionnel à la liberté prise par rapport à l'imprimé, un support qui, il faut le rappeler, est dans ce cas une importation du colon hollandais. Ainsi des cercles littéraires se forment, puis disparaissent de manière très volatile à travers toute l'Indonésie. Des aèdes contemporains s'y produisent mêlant matériel traditionnel et création originale. Parfois leurs narrations paraissent dans des journaux à grand tirage, mais rares sont les livres tels que nous les entendons ${ }^{119}$.

Nous avons vu au chapitre 3 l'importance, désormais, sous nos latitudes, de la performance pour la littérature, ainsi que la porosité et la collectivisation des « je » d'auteurs dans la culture digitale ${ }^{120}$. Les remarques de Derks font donc absolument mouche, lorsqu'il décrit ainsi les transmissions et lieux de performances littéraires indonésiens :

L'utilisation de la métaphore du mycélium a non seulement l'avantage de rendre compte de la multi-centralité des littératures indonésiennes en Malaisie ; elle met aussi en lumière la conviction que cette littérature doit

\footnotetext{
115 Wasserman - Gurry, A New Approach to Textual Criticism, p. 27.

116 Greetham, «Phylum-Tree-Rhiozome », p. 106 ; D. Deleuze - F. Guattari, Rhizome, Editions de Minuit, 1976 ; réédité dans G. Deleuze - F. Guattari, Capitalisme et Schizophrénie, Editions de Minuit, 1980. ture in Maly », Journal of Southeast Asian Studies 32 (2001), p. 367-384.

118 Derks, « A literary Mycelium », p. 368.

119 Touati, « Pourquoi éditer », p. 10.

120 Voir chapitre 3, point 3.2.
} 
être considérée comme un système vivant: un fugace continuum qui pulse et respire, dans les termes duquel la vie littéraire pourrait être un champignon à diverses places et par les diverses manières d'activités littéraires diversifiées qui reflètent toutes, d'une manière ou d'une autre, une forte orientation orale ${ }^{121}$.

Ce type de relation à la textualité et à la littérarité, emprunte d'oralité, mise en exergue dans la culture digitale, se retrouve maintenant jusque dans les travaux littéraires sous nos latitudes, comme par exemple dans cette description de Baptiste Gaillard :

Comme la chrysalide pour les chenilles, le texte est une vaste usine de mutations. Il se déploie par capillarité et grandit par le milieu. Sa matière en évolution est régulièrement découpée, déplacée, réorganisée. Certains blocs se divisent pour former de nouveaux fragments, alors que d'autres entre eux s'agrègent. Du singulier ponctue l'itération des motifs, comme du solide restant dans un bain de macération : la délimitation est incertaine entre ce qui est déjà, et ce qui résiste encore ${ }^{122}$.

Si, comme on l'a vu avec François Jouen au chapitre 2, l'intelligence artificielle nous demande de travailler à de nouvelles représentations qui n'ont pas forcément de lien au biologique, il en va tout autrement de la textualité, dont ces divers exemples montrent la solidarité avec les métaphores du vivant. La métaphore du mycélium est parlante ; on pourrait la compléter avec une propriété intéressante du blob, sa manière de transmettre la connaissance :

Ni animal, ni plante, ni champignon, le «blob» (Physarum polycephalum) est un curieux être rampant composé d'une unique cellule géante. Bien que dépourvu de cerveau, il est capable d'apprendre de ses expériences. [...] Imaginez que vous ayez la capacité de fusionner temporairement avec un autre individu et quà l'issue de cette expérience, vous ayez acquis toutes ses connaissances. Eh bien, chez les blobs, c'est possible! [...] A présent, [les chercheurs] montrent qu'un blob ayant appris à

121 Derks, «A literary Mycelium », p. 373: «The use of the metaphor of the mycelium not only has the advantage of accounting for the multi-centeredness of Indonesian literatures in Malay, it also emphasises the conviction that this literature should be seen as a living system : a pulsating, breathing continuum of transience in terms of which literary life may mushroom in various places and by means of a great variety of literary activities that all somehow reflect a strong oral orientation ».

B. Gaillard, Un domaine des crépuscules, Hippocampe, 2017, page de couverture. 
ignorer le sel peut transmettre son apprentissage à l'un de ses congénères, tout simplement en fusionnant avec lui !123

Surprenante et pas très claire, la métaphore du blob et de son mode d'apprentissage ? Certes, mais ce flou de l'inconnu peut peut-être justement nous être utile pour décrire les nouvelles expériences de la textualité et de la littérature digitales, où l'oralité et les images viennent redistribuer les cartes des réseaux de transmission. Le blob représente un mode de fonctionnement décentralisé par excellence, tels les brins d'ADN décrits par Kiefer comme ne cessant « de se référer à leur propre structure, [...] organisés en métatextes, boucles étranges et enchevêtrements superposés ${ }^{124}$. Il sera sans doute plus simple d'interroger nos représentations métaphoriques de la textualité que celles de l'ADN, mais il n'est en tous cas pas surprenant que ces remises en question s'annoncent en parallèle, au vu de la solidarité entre textes et corps, soulignée tout au long de ce volume et développée au point 2.3.1 ci-dessous.

Je propose donc de garder en tête la métaphore diffuse du blob, associée à celle du mycélium, pour aborder au point 2 la question des corpus et corpora textuels. Elle assume de couper avec une représentation linéaire, mais stimule les nouvelles représentations des contacts et influences entre traditions. Auparavant, le point 1.4 va nous permettre de faire un tour d'horizon des théologiens qui se posent la question du statut du texte biblique dans la culture digitale.

\subsection{Discuter le statut du texte biblique dans la culture digitale}

Les points 1.1 et 1.2 ont présenté les travaux de différents chercheurs en sciences bibliques ou théologie qui se sont penchés sur la culture digitale, mais sans estimer que celle-ci pouvait influencer la perception du statut du texte biblique. En revanche, nous avons vu au chapitre 1 que le rapport de la FE Ps Sola lectura? reliait la question des Ecritures digitales à celle de la canonicité, via leur « émancipation » hors de l'écrit ${ }^{125}$.

De fait, ce questionnement sur la résistance canonique des Ecritures digitales se mène plutôt à partir des usages et pratiques, telles les applications, quà partir de l'édition même du Nouveau Testament. Comme le décrit Tim Hutchings, « dans de nombreuses églises et groupes d'études de la Bible, tout au moins en Grande-Bretagne et aux Etats-Unis, il est désormais courant de voir

123 CNRS, «Le 'blob’ capable d'apprendre... et de transmettre ses apprentissages », Communiqué de presse 21 décembre 2016, <http://www2.cnrs.fr/presse/communique/4837.htm>.

124 Kiefer, « Nouveaux concepts », p. 816.

125 FEPS, Sola lectura?, p. 10-11 ; cité au chapitre 1, p. 20-21: «l'Ecriture en format électronique est plus interactive et moins canonique ; elle n'est plus l'Ecriture sainte codifiée sous forme imprimée, mais fait partie d'un processus continu de communication ». 
utilisés des téléphones mobiles et tablettes durant les cérémonies, plutôt que des Bibles imprimées. [...] Les éditeurs ont commencé à augmenter la Bible de ressources multi-média, promettant à l'utilisateur de l'aider à s'engager plus profondément et plus fréquemment avec le texte ${ }^{126}$. Cette évolution a depuis longtemps suscité des craintes, notamment dans les milieux théologiques évangéliques : les Ecritures allaient-elles se liquéfier sur internet, et perdre leur canonicité avec leur couverture ? Les enthousiasmes ont également été à la hauteur des craintes, comme le montrent les quelques exemples suivants.

En 1990, Phil Mullins se demandait déjà si le support d'écriture digital allait être capable de maintenir la frontière entre textes bibliques et sources extérieures ${ }^{127}$. Tom Beaudoin est connu pour avoir émis l'une des plus pessimistes assertions en 1998, relevée par Tim Hutchings : « Il est impossible d'avoir un texte sacré dans le cyberespace, une cyber-bible est toujours errante (wandering) » ${ }^{128}$. Quant à Rachel Wagner, elle estimait en 2012 que les chrétiens ont désormais à décider pour eux-mêmes de ce qui doit être inclus ou non dans le Nouveau Testament, et que la Bible elle-même devenait fluide ${ }^{129}$. En même temps, Hutchings a rappelé que plusieurs analyses des discours d'autorité, religieux ou politiques, ont montré que les leaders politiques ou religieux savaient très bien retomber sur leurs pieds dans la culture digitale pour maintenir un cadre interprétatif ${ }^{130}$.

Pour avoir une idée plus précise de la problématique, on peut notamment observer ce qui se passe au sein des deux applications bibliques qui connaissent le plus grand succès en termes d'utilisateurs, YouVersion et GloBible ${ }^{131}$, promues par des mouvements chrétiens évangéliques ${ }^{132}$. YouVersion a été fondée par Life.Church, qui n'est pas « une communauté indépendante en ligne, mais le ministère en ligne d'une seule entité ecclésiastique fondée aux Etats-

126 Hutchings, «Design and the digital Bible», p. 205: «in many churches and Bible study groups, at least in Britain and the United States, it is now common to see mobile phones and tablets used during services instead of printed Bibles. [...] Publishers have begun to augment the Bible with multi-media resources, promising to help the user achieve a deeper and more frequent engagement with the text $»$.

127 P. Mullins, «Sacred Text in an Electronic Age», Biblical Theology Bulletin 20 (1990/3), p. 99-106 ; ici p. 105.

128 T. Beaudoin, Virtual Faith. The Irreverent Spiritual Quest of Generation X, Jossey-Bass, 1998, p. 126 ; cité par Hutchings, « Design and the digital Bible », p. 205.

129 R. Wagner, Godwired:Religion, Ritual and Virtual Reality, New York, Routledge, 2012, p. $22-$ 23 .

130 Hutchings, « Design and the digital Bible», p. 206.

131 Youversion, <https://www.youversion.com/> ; GloBible, <https://globible.com/>.

132 Voir aussi Hutchings, « E-reading and the Christian Bible». 
Unis en 1997 »; en 2009, elle comptait treize emplacements géographiques ${ }^{133}$. A lire l'analyse qu'en fait Hutchings, la Bible n'est absolument pas en train de « s'évanouir » ou de devenir « liquide » dans ces applications. De fait, il démontre qu'elles maintiennent un cadre d'interprétation évangélique très carré, exploitant toutes les possibilités multi-média :

Ces produits offrent de larges bibliothèques, avec des options audio et multi-média et des milliers de textes à y choisir, mais leurs portfolios ne sont pas infinis. Les contenus sont soigneusement sélectionnés, tout comme le sont les options de l'utilisateur pour naviguer dans la bibliothèque. Parfois, comme déjà signalé, le produit numérique peut même agir à l'encontre de l'indépendance de l'utilisateur, apportant un conseil, réprimandant l'entêté, et utilisant les techniques de persuasion technologique pour former à de nouvelles habitudes d'engagement textuel [...]. Mon argumentation démontre que les bailleurs de fonds, les designers et responsables du marketing de certaines Bible numériques sont en train d'essayer avec opiniâtreté de promouvoir une attitude évangélique traditionnelle envers la Bible, mais une recherche subséquente sera nécessaire pour évaluer les conséquences de l'adoption à large échelle d'un texte digitalisé dans les communautés religieuses ${ }^{134}$.

Dans la foulée de cette analyse d'Hutchings, je peux moi-même témoigner d'une limitation des possibilités dans l'application YouVersion, depuis un article que j'ai publié en 2017. J'y indiquais qu'il était possible d'être informé sur la quantité de versets ou chapitres bibliques lus ou écoutés en audio, par pays $^{135}$. Est-ce que l'importance des chapitres bibliques écoutés a pu sembler

133 Hutchings, Creating Church, <https://online.vitalsource.com/books/9781136277498/epub cfi/6/32!/4[cho8]/8@0:31.3>.

134 Hutchings, «Design and the digital Bible », p. 215-216: «These products offer extensive libraries, with audio and multi-media options and thousands of texts to choose from, but their portfolios are not infinite. Contents are carefully chosen, as are the user's options for navigation through the library. At times, as indicated above, the digital product can even go against the user's independence, offering advice, reprimanding the wayward, and using the techniques of persuasive technology to form new habits of textual engagement. [...] My evidence demonstrates that the funders, designers, and marketers of some digital Bibles are trying hard to promote a traditional Evangelical attitude to the Bible, but further research will be needed to evaluate the consequences of widespread adoption of digital text within religious communities ».

135 Clivaz, «Die Bibel », p. 57: «Ein besonderes Phänomen scheint die Wiederkehr der Mündlichkeit zu sein. Am 15. April 2017 war auf YouVersion zu erfahren, dass dessen Benutzer in Indien 166 schriftliche Verse geteilt haben, dass in Ägypten 3525 Audio-Kapitel gehört wurden, und 94 in der Ukraine. Am selben Tag wurden in Schweden 25 schriftliche 
un élément menaçant le rapport classique du croyant au texte écrit ? En tout cas, une telle consultation n'est en ce moment plus possible sur YouVersion, et les versions audio des chapitres bibliques ne sont pas annoncées dans les

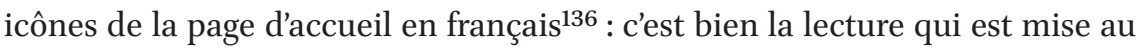
premier plan.

Comme le montre le début de ce point 1.4, la question de la résistance du corpus des Ecritures bibliques à la liquidité des textes sur internet rencontre rapidement la question des communautés chrétiennes, utilisatrices de ce corpus et du cadre interprétatif qu'elles posent, reflétant la théorie des communautés interprétatives de Stanley Fish ${ }^{137}$. J'avais pour ma part aussi soulevé la question du devenir du Nouveau Testament sur internet qui pourrait se retrouver être un « tout petit livre » perdu sur le Web, selon l'expression d'Ap 10, 2.9${ }^{10}{ }^{138}$. L'analyse qu'Hutchings a fait de YouVersion et GloBible tendrait à montrer que la question s'avère au bout du compte plus sociologique que textuelle ou théologique, et souligne que le cadre des communautés interprétatives maintient globalement la stratégie canonique à l'œuvre. Toutefois, la lecture de la première monographie sur la Bible dans la culture digitale, publiée par Siker, permet de repérer la notion de corpus biblique comme un défi à aborder, ce que je ferai au point 2. J'y montrerai notamment les liens entre canonicité et évolution des supports d'écriture.

L'ouvrage de Jeffrey Siker marque de manière claire le tournant de la culture digitale dans les sciences bibliques. J'en présente ici les points principaux, en renvoyant pour une critique complète au compte-rendu anglais publié dans la $\mathrm{RBL}^{139}$. La monographie de Siker parvient à la fois à présenter les nouveaux usages et habitudes induits par les Bibles digitales (chapitres 1, 4 et 5), une synthèse de la Bible et des évolutions technologiques (chapitre 2), un historique des Bibles digitales (chapitre 3), et une analyse du statut de la Bible dans la culture digitale (chapitre 6), dans les médias sociaux et linformatique (chapitres 7 et 8), puis une ouverture sur les défis du futur (chapitre 9).

Dans sa discussion du statut de la Bible digitale au chapitre 6, Siker passe en revue de manière exhaustive les conséquences positives et négatives de

Verse geteilt, aber im selben Land 2532 Kapitel der Bibel angehört. Diese Beobachtungen fügen sich zum generellen Eindruck einer Wiederkehr der Mündlichkeit in der westlichen Kultur».

136 Les langues et les traductions de la Bible, <https://www.bible.com/fr/languages >.

137 S. Fish, Is There a Text in This Class? The Authority of Interpretive Communities (Literary Criticism), Harvard University Press, 1982. Pour une présentation, voir Clivaz, L'ange et la sueur de sang, p. 163-165.

138 Clivaz, « New Testament in Digital Culture ».

139 Clivaz, « Review of Jeffrey S. Siker ». 
l'aspect « liquide » du texte biblique digitalisé. De manière originale, il y soulève aussi la question de la traduction, qui est un point nodal de la réception biblique, et qui est tout sauf en passe d'être résolu par l'informatique ${ }^{140}$. Mais dans l'introduction, il s'était aussi montré inquiet à ce même propos : « la disponibilité immédiate de tant de traductions dans la forme digitale conduit à une certaine déstabilisation du texte biblique $»^{141}$. L'auteur exprime ailleurs des soucis similaires, par exemple : « la Bible déliée sur écran ne se prête pas d'elle-même à une prise de conscience immédiate d'une forme particulière de la Bible, canonique ou autre. Dans cette perspective, survoler la Bible sur des écrans conduirait nécessairement à sous-estimer la compréhension de la Bible dans son cadre canonique $»^{142}$. Tout comme dans le rapport de la FEPS, on aurait ici donc la crainte assumée d'un déficit canonique des Ecritures digitales.

Toutefois, Siker n'intègre pas du tout les travaux de Tim Hutchings sur le maintien des discours d'autorité religieuse dans la culture digitale, et cela alors même qu'il commente largement l'application YouVersion. Aussi manque-t-il les informations de cette analyse sur la présence forte de cadres conceptuels chrétiens dans les produits bibliques numérisés. Cette omission est peut-être tout simplement due au fait qu'il participe lui-même à ces cadres. En effet, son lectorat est clairement assumé comme celui des communautés chrétiennes, des lecteurs convaincus que la «Bible n'est pas juste un livre unifié, mais le Livre des livres $»^{143}$. Une telle conviction rappelle le ton de Cadbury ou van Unnik ${ }^{144}$, et ne manquera pas de surprendre le lecteur de culture européenne. Mais elle pointe exactement sur la grand oubliée de toutes les discussions évoquées dans cette première partie : la notion de corpus.

Si Siker parle du «Livre des livres », nous avons vu aussi que Wasserman et Gurry avaient pour point de départ, et sans remise en question, l'ensemble des vingt-sept livres du Nouveau Testament ${ }^{145}$. D'autres voix, comme le rapport Sola lectura? ou Rachel Wagner, pressentaient les possibles transformations de

$140 \quad$ Siker, Liquid Scripture, p. 83-91.

141 Siker, Liquid Scripture, p. 5.

142 Siker, Liquid Scripture, p. 69 : « The unbound Bible on a screen does not lend itself to an immediate awareness of any particular shape of the Bible, canonical or otherwise. From this perspective skimming the Bible on screens would necessarily seem to undermine understanding the Bible in its canonical frame ».

143 Siker, Liquid Scripture, p. $9:$ : Even though its contents range far and wide both in terms of historical context and theological vision, when the words 'The Bible' are embossed in gilded lettering on its leather cover and spine it becomes not just a unified book, but the Book of all books. It is no wonder that the Bible continues to be the best- selling book of all time, year after year, version after version, translation after translation ».

144 Voir l'Introduction, p. 12.

145 Voir p. 177 ci-dessus. 
ce corpus, sans toutefois en explorer la notion. C'est donc bel et bien à cet endroit où les Ecritures font corps qu'il faut évaluer l'impact de la culture digitale.

Comme on l'a vu au point 1, les spécialistes du NT et les théologiens soit continuent à considérer les vingt-sept livres du Nouveau Testament comme un corpus de textes inébranlable, soit travaillent la problématique du corps des Ecritures digitales en fonction de la question théologique et historique du canon. Je propose, pour adopter le point de vue le plus judicieux possible sur cette question, de commencer par observer ce qu'il advient de la notion de corpus textuel dans l'édition digitale en général. De fait, la question des corpus ou collections de textes y devient une thématique de plus en plus centrale.

Le point 2.1 va permettre d'observer que la matérialité même du support d'écriture digitale reconfigure notre perception de la notion de corpus textuel, qui évolue vers celle de collection multimodale. Le point 2.2 revisitera la thématique du canon des Ecritures en fonction de la matérialité du support d'écriture. Le point 2.3 déploiera pour conclure une réflexion sur le corps et l'écriture, et le corps des Ecritures en régime digital. Il évaluera, pour ces dernières, les conséquences de l'hypothèse présentée au chapitre 3 pour l'écriture digitale en général, et aboutira à cette proposition : c'est via la multimodalité que les Ecritures digitales sont appelées à nouer un lien novateur à la corporéité.

\subsection{Du corpus textuel à la collection digitale}

Comment désigner ce que produit l'édition digitale ? Elena Pierazzo rappelle qu'en 2009, Kenneth Price a clairement décrit la complexité de la situation actuelle des éditions digitales qui produisent une « édition », un « projet», une « archive», ou une « collection de recherche thématique digitale», qu'il a proposé de nommer finalement et non sans ironie « arsenal » ${ }^{146}$. Elle commente ainsi cette diversité nominale : « cette variété de noms manifeste clairement la transformation de nature des productions d'actes éditoriaux $[\ldots]$ : premièrement, elles se présentent sous la forme d'un site web, soit un artefact digital et physique qui est extensible indéfiniment; deuxièmement, ces productions

146 Pierazzo, Digital Scholarly Editing, p. 193 ; elle se réfère à K. M. Price, « Edition, Project, Database, Archive, Thematic Research Collection: What's in a Name? », DHQ 3 (2009/3), $<$ http://www.digitalhumanities.org/dhq/vol/3/3/000053/000053.html $>$. 
sont par nature collaboratives ${ }^{147}$. Et notre auteur de relever l'extension quasi infinie de ce que le terme de digital library peut désormais recouvrir ${ }^{148}$.

La problématique est ainsi posée d'entrée : parce qu'un lieu d'édition digitale peut par définition accueillir divers types de matériel, être extensible à l'infini et se déployer de manière collaborative, la délimitation même d'un corpus est remise en question. Comme l'exprime Sarah Mombert, « les éditions digitales sont déconnectées de la valeur institutionnelle des livres. [...] Elles semblent vouloir entrer dans l'ère de la collection ${ }^{149}$. Le principe d'extension d'une collection, ou d'une dynamic library, s'observe aisément dans le projet européen Sharing Ancient Wisdoms (sAWs), consacré à rassembler des «collections d'idées et d'opinions - des dires de la Pythie à de brefs passages de longs textes philosophiques - qui ont façonné le genre antique de la littérature de sagesse. On trouve de telles collections dans toutes les cultures du MoyenOrient et de l'Europe ${ }^{150}$.

Si cinq groupes de textes forment la base de ce projet ${ }^{151}$, on imagine aisément qu'il pourrait être complété presqu'à l'infini. Comme le relève Mombert, c'est au bout du compte le temps et les ressources financières qui finissent par déterminer la délimitation du contenu d'un projet éditorial digital ${ }^{152}$. Par-delà ces limitations concrètes, un projet tel que sAws impressionne par sa capacité d'intégration - ou de réintégration - de textes fragmentaires et qui étaient hors circuit des chemins principaux de la recherche.

Ce travail de réintégration du fragment par la collection d'édition digitale pourrait indiquer un tournant important dans l'histoire des représentations. En effet, alors que la culture imprimée est en plein développement et va atteindre son apogée, la littérature des 18e et 19e siècles va développer des représentations littéraires des topos du manuscrit perdu et retrouvé, ainsi que du fragment. Célèbre au moins depuis Don Quichotte, ce topos s'est vu consacré un ouvrage collectif en 1999, édité par Jan Herman, Fernand Hallyn et Kris Pee-

147 Pierazzo, Digital Scholarly Editing, p. 193: «Clearly, this variety of names is a manifestation of the changing nature of the outcomes of editorial acts [...] : firstly, that it is a website, a digital and physical artefact which is infinitely extensible ; secondly its collaborative nature ».

148 Pierazzo, Digital Scholarly Editing, p. 196-198.

149 S. Mombert, «From Books to Collections. Critical Editions of Heterogeneous Documents », dans A. Apollon - C. Bélisle - P. Régnier (éd.), Digital Critical Editions (Topics in the Digital Humanities), University of Illinois Press, 2014, édition Kindle ; ici l. 5143.

$150 \quad$ Sharing Ancient Wisdoms, <http://www.ancientwisdoms.ac.uk/about/>.

151 Sharing Ancient Wisdoms, <http://www.ancientwisdoms.ac.uk/library/>.

152 Mombert, « From Books to Collections », édition Kindle, l. 5248. 
ters ${ }^{153}$, où prend place notamment un article d'Emmanuèle Baumgartner soulignant les consonances entre manuscrit et corps ${ }^{154}$. Autre exemple de ce topos : l'édition et travaux de François Rosset sur le Manuscrit trouvé à Saragosse de Jean Potocki ${ }^{155}$.

Quant au fragment, il est illustré de manière éminente dans le roman The Castle of Otranto d'Horace Walpole ${ }^{156}$, par ailleurs créateur de la notion de sérendipité, comme nous l'avons vu ${ }^{157}$. Mais l'entrée en scène du fragment dans la réflexion littéraire, philosophique et épistémologique a lieu au 17e siècle avec la publication du livre atypique de Jean de La Bruyère, Les Caractères, composé de 418 fragments pour l'édition de 1688 et 1120 pour l'édition de ${ }_{1696} 6^{158}$. Dans un commentaire incisif, Pascal Quignard introduit à ce qu'a signifié la publication de cet ouvrage du temps de La Bruyère, ses détracteurs lui reprochant qu'un « livre fait de fragments n'est pas un livre. [...] Ce n'est qu'un amas de pièces détachées ». La Bruyère lance ainsi un genre en 1688 qui est un « système délibéré de la fragmentation volontaire dans la prose française », même si d'autres auteurs s'y essaient également ${ }^{159}$. Le fragment contient de la violence, est un «cancer qui corrompt l'unité d'un corps et qui le désagrège $»^{160}$, explique Quignard en rappelant aussi les couleurs étymologiques $\mathrm{du} \ll$ fragment $»:$

Les mots latins de fragmen, de fragmentum viennent de frango, briser, rompre, fracasser, mettre en pièces, en poudre, en miettes, anéantir. En grec, le fragment, c'est le klasma, l'apoklasma, l'apospasma, le morceau détaché par fracture, l'extrait, quelque chose d'arraché, de tiré violemment. Le spasmos vient de là : convulsion, attaque nerveuse, qui tire, arrache, disloque ${ }^{161}$.

153 J. Herman - F. Hallyn - K. Peeters (éd.), Le topos du manuscrit trouvé. Actes du colloque international Louvain-Gand, 22-23-24 mai 1997 (Bibliothèque de l'information Grammaticale 40), Peeters, 1999.

154 E. Baumgartner, «Du manuscrit retrouvé au corps retrouvé », dans J. Herman - F. Hallyn - K. Peeters (éd.), Le topos du manuscrit trouvé, p. 1-14.

155 J. Potocki, Manuscrit trouvé à Saragosse, F. Rosset (éd.), Peeters, 2006 ; F. Rosset (éd.), Entretiens sur le Manuscrit trouvé à Saragosse, Etudes de Lettres, 2012, <https://journals. openedition.org/edl/408>.

156 H. Walpole, The Castle of Otranto, London, William Bathoe, 1766.

157 Voir chapitre 3, p. 110-111.

$15^{8}$ J. de La Bruyère, Les Caractères, R. Garapon (éd.), Classiques Garnier, 1962 ; P. Quignard, Une gêne technique à l'égard des fragments, Fata Morgana, 1986, p. 19.

159 Quignard, Une gêne technique, p. 37.

160 Quignard, Une gêne technique, p. 23.

161 Quignard, Une gêne technique, p. 33. 
Le fragment, par définition, a donc toujours été ce qui gênait dans un corpus de textes, ce qui ne pouvait y prendre place, faisant violence à la linéarité ordonnée du déroulement du texte. Ce rejet du fragment dans la culture imprimée est précisément mis en lumière par le projet numérique sAws, qui vise à rassembler ce qui n'a pas trouvé place ailleurs et démontre le vide existant pour ce type de textes en pièces détachées. C'est exactement ici qu'intervient ce que nous pouvons qualifier de renversement dans la culture digitale. En tant que collection digitale, saws offre la possibilité de faire revenir les marges vers le centre, ou simplement de les rendre accessibles. Une collection digitale permet donc de déconstruire la canonicité classique instituée dans la littérature, « canonicité » s'entendant ici au sens large de ce terme. Mombert décrit ainsi ce trait de la collection digitale :

Pour les textes non canoniques (par exemple les documents qui n'ont pas été jugés dignes jusqu’à maintenant d'être réédités avec un apparat critique et ont été maintenus hors du circuit traditionnel de livres connus), [...] la technologie digitale représente non seulement l'occasion d'être sauvés des ravages du temps, mais elle signifie aussi la fin d'un statut éditorial marginal ${ }^{162}$.

En termes symboliques, pour se représenter l'effet d'une collection digitale sur les textualités, on pourra dire qu'au bout du compte, elle les défragmente, elle les réinsère dans un lieu et un environnement, à l'image de l'opération informatique de défragmentation d'un disque :

La défragmentation d'un disque est le processeur de consolidation de données fragmentées dans un volume (tel que disque dur ou instrument de stockage), de sorte à ce qu'il fonctionne plus efficacement. Une fragmentation se produit dans un volume avec le temps lorsque vous sauvegardez, modifiez ou détruisez des dossiers ${ }^{163}$.

162 Mombert, «From Books to Collections », édition Kindle, l. 5128 : «From the viewpoint of non-canonical texts (e.g., documents that until now had been deemed not worthy of reeditions with a critical apparatus and were kept out of the traditional circuit of learned books) [...] digital technology represents not only the opportunity of being salvaged from the ravage of time but also the end of a marginal editorial status ».

163 What is disk defragmentation?, <https://www.disktuna.com/da-disktuna/> : « Disk defragmentation is the process of consolidating fragmented data on a volume (such as a hard disk or a storage device) so it will work more efficiently. Fragmentation happens to a volume over time as you save, change, or delete files ». 
L'opération de défragmentation d'un disque informatique nous offre ainsi une métaphore pour exprimer ce qu'il arrive aux fragments textuels lorsqu'ils intègrent une édition digitale au sein d'une collection : les voici défragmentés, réordonnées, reclassés et remis en réseaux avec d'autres données culturelles. Cette opération, qui a un effet très important quant au contenu, se présente d'abord essentiellement sous ses critères pragmatiques de matérialité numérique, comme le souligne Franz Fischer, dans une étude sur les corpus digitaux de textes latins ${ }^{164}$. Parmi les quatre critères qu'il donne pour définir une édition digitale, seul le quatrième est philologique. L'attention va ici au marquage, aux métadonnées, à la fonction recherche, à la fonction d'indexation, aux hyperliens, à la citabilité165. Tout comme Elena Pierazzo, Fischer souligne l'aspect open-ended d'une édition digitale, qui prend la figure d'une encyclopedic knowledge que Fischer décrit ainsi :

Les éditions digitales qui participent à un corpus ne peuvent pas et ne doivent pas être complètement inclusives. Au contraire : une des caractéristiques des éditions digitales est de surmonter les limitations de la publication elle-même via l'intégration, ou même plus important, via les liens aux ressources externes ${ }^{166}$.

Pour Fischer, c'est donc via la mise en réseaux des ressources qu'on étend les limites d'une édition digitale, et non pas en la concevant comme un « grand tout », à la mode de la bibliothèque arabophone Shamela, présentée en 2.2.2 ci-dessous ${ }^{167}$. Si donc la matérialité même des éditions digitales - capables de s'étendre sans autres limites que celles du temps et des ressources à disposition, collaboratives et défragmentant les classifications littéraires canoniques - les fait sortir de la définition de corpus textuels, une autre terminologie devient nécessaire pour désigner ce qu'elles produisent. Mombert soutient le choix de « collection », un terme assez souple pour intégrer une documentation et des objets digitaux hétérogènes, comme elle le définit :

164 F. Fischer, « Digital Corpora and Scholarly Editions of Latin Texts: Features and Requirements of Textual Criticism », Speculum 92 (2017/1), p. 267-287; <https://www.journals. uchicago.edu/doi/abs/10.1086/693823>.

165 Fischer, «Digital Corpora », p. 278-279 et 284-286.

166 Fischer, « Digital Corpora », p. 281: «Digital editions as part of a corpus cannot and should not be all inclusive. To the contrary : a characteristic of digital editions is the overcoming of the limitations of the publication itself through integration of or, here even more importantly, through linkage to external resources ».

167 Q. Assef, "Bibilothèque numérique "Shamela" : 10'0oo ouvrages en langue arabe", $<$ https://ifpo.hypotheses.org/683> ; voir p. 209 ci-dessous. 
Le terme de « collection » a l'avantage d'être historiquement lié aux domaines concernés par l'édition critique : culture et science. [...] Dans le domaine de l'édition digitale, où l'usage du terme de « collection » n'est pas encore fixe, je voudrais proposer cette définition minimale : un ensemble d'objets digitaux susceptible d'évolution, et qui a l'intention de produire une signification ${ }^{168}$.

Cette définition souple permet d'intégrer ce que l'auteur considère comme l'un des points les plus cruciaux du changement de régime : « la relation entre textes et images, appréhendée entre deux objets fondamentalement hétérogènes $»^{169}$. Si Mombert travaille ce point dans son article en l'illustrant d'exemples, elle n'envisage toutefois pas encore la question des sources sonores qui peuvent être intégrées dans les collections digitales, ni du point de vue du contenu, ni du point de vue des défis numériques. Mais elle pose bel et bien la base du critère de multimodalité des sources dans sa démonstration pour présenter la notion de « collection » digitale, appelée vraisemblablement à remplacer celle de corpus textuels.

Le phénomène s'accentuera d'autant plus si, insérée dans la « collection digitale », l'édition textuelle digitale se prête au jeu du linked open data (LOD ) $)^{170}$. Ce sera le cas lorsqu'on déposera par exemple des textes encodés sur un dépôt ouvert, tel Nakala, un service assuré par le consortium français Huma-Num ${ }^{171}$. Toutefois, ni Elena Pierazzo, dans sa monographie dense sur l'édition digitale, ni le collectif bien informé d'Apollon, Bélisle et Régnier, n'abordent la thématique du LOD, et pour cause ${ }^{172}$. Né au creuset du web sémantique lancé par Tim Berners Lee ${ }^{173}$, le LOD représente clairement une étape qui dépasse la notion classique d'édition textuelle pour considérer les textes comme des objets

168 Mombert, «From Books to Collections », édition Kindle, l. 5186: « The term 'collection' has the advantage of being historically linked to the domains concerned with critical edition : culture and science. [...] In the digital edition domain, where the use of the term 'collection' is still not fixed, I would propose this minimal definition : a potentially evolutionary set of interlinked digital objects, with the intention of producing some meaning ».

169 Mombert, « From Books to Collections », édition Kindle, 1. 5157.

170 L'article de Wikipedia en français sur ce thème ne traduit pas l'expression anglaise (https://fr.wikipedia.org/wiki/Linked_open_data). Je remercie le reviewer anonyme qui m’a suggéré d'ajouter une réflexion sur le LOD.

171 Voir Nakala, <https://www.nakala.fr/> ; Huma-Num, <https://www.huma-num.fr/>.

172 Pierazzo, Digital Scholarly Editing; Apollon - Bélisle - Régnier (éd.), Digital Critical Editions.

173 Voir pour la mise en place et le développement du web sémantique et du LOD $:<\mathrm{https} / / /$ www.w3.org/DesignIssues/LinkedData.html > ; https://www.w3.org/TR/owl2-over view/>. 
pouvant s'associer à différents types d'objets, de tous genres. Les archéologues, par exemple, ont rapidement compris l'intérêt du LOD pour mettre en liens les objets qu'ils étudient ${ }^{174}$. Pour le dire autrement, le LOD accentue encore plus le passage du corpus textuel, de l'édition digitale, à la collection digitale, multimodale.

Comme le point 2.3 le soulignera, la solidarité entre corps et textes est séculaire : il avait donc été absolument logique et naturel de parler de « corpus textuels ». Si l'on passe à un régime d'ensemble d'objets culturels où s'intègrent les images, les sons avec les textes, reliés par LOD, on quitte cette solidarité séculaire entre le corps et le texte. Un nouveau vocabulaire, de nouvelles représentations, sont dès lors requis. En retour, le matériel culturel numérique devient libre de contracter de nouvelles relations au corps, à commencer par le stockage multimédia opéré dans l'ADN et présenté au chapitre 3, en écho au roman prophétique de Bradbury Fahrenheit 451 et au vœu de Derrida, rêvant de « vivre sans papier », «la 'vraie vie', [le] vivant de la vie » ${ }^{175}$. Nous approfondirons ces aspects au point 2.3, en testant les possibles pour les Ecritures digitales, dont l'aspect canonique peut être montré au travers d'une histoire de leur support matériel d'écriture, comme le point 2.2 va le présenter.

\subsection{Christianisme et corpus textuels}

Comme nous l'avons vu au point 1, le discours des spécialistes du NT et des théologiens sur le corpus biblique dans la culture digitale oscille entre deux attitudes. Ce corpus est d'un côté clairement réaffirmé comme base de travail, par exemple dans l'introduction à la С BGM de Wasserman et Gurry, qui pose la prédication dominicale comme l'un des buts de leur ouvrage ${ }^{176}$. Le cadre interprétatif canonique est même on ne peut plus clairement acquis dans une application comme YouVersion, qui a un cadre interprétatif ferme, comme on l'a vu avec Hutchings ${ }^{177}$. D'un autre côté, plusieurs théologiens ressentent la crainte diffuse que la culture digitale n'attente à la canonicité des Ecritures, du rapport Sola lectura? de la FEPS à Jeffrey Siker, en passant par Rachel Wagner $^{178}$. En revanche, aucun d'entre eux ne semble avoir pris acte de l'évolution

174 Voir par exemple L. Isaksen, M. Kirk Martinez, N. Gibbins, G.Earl and S. Keay, « Linking Archaeological Data », dans Making History Interactive. Computer Applications and Quantitative Methods in Archaeology. Proceedings of the 37th International Conference, Williamsburg, Virginia, United States of America, March 22-26, B. Frischer, J. Webb Crawford, and D. Koller (éd.), Archaeopress, 2010, p. 130-136.

175 Voir chapitre 3, p. 129.

176 Wasserman - Gurry, A New Approach to Textual Criticism, p. 9 ; cité en note p. 177 ci-dessus.

177 Hutchings, « Design and the digital Bible », p. 215-216; cité p. 193 ci-dessus.

178 Voir le point 1.4 de ce chapitre. 
en cours présentée au point 2.1, ou y avoir réfléchi : la mutation progressive des corpus de textes vers des collections d'objets digitaux.

Le défi est donc dans ce point 2.2 d'arriver à articuler la question de la canonicité des Ecritures, ou des catégories de textes chrétiens anciens, avec celle de la matérialité du support d'écriture, qui est au cœur de la transition du corpus textuel à la collection digitale. Nous commencerons par rappeler le contexte culturel de quelques points du débat récent autour de ces catégories de textes, puis nous montrerons que la question du support matériel d'écriture est l'un des facteurs importants, mais sous-estimé, voire négligé, de ce débat ${ }^{179}$.

Le débat actuel sur les catégories de textes chrétiens anciens dans son contexte culturel

Comme nous l'avons constaté tout au long de cet essai, le débat académique sur les catégories de textes chrétiens anciens, entre canon du Nouveau Testament et textes qui n'appartiennent pas à ce canon, reste encore largement influencé par des enjeux confessionnels, ou tout au moins par un positionnement culturel chrétien. C'est ainsi que François Bovon, revendiquant une posture de théologien « nourri aux pieds de Karl Barth » ${ }^{180}$, a proposé d'établir l'existence d'une troisième catégorie textuelle, située entre les textes chrétiens canoniques et apocryphes : les textes « utiles à l'âme », $\psi \mho \chi \omega \varphi \varepsilon \lambda \hat{\varsigma} \varsigma$, psuchôphélès. Il la définit ainsi :

Je crois qu'il y avait une troisième destinée : certains livres n'ont été ni rejetés comme apocryphes, ni admis comme canoniques, mais étaient considérés comme utiles et profitables à l'âme ( $\psi \cup \chi \omega \varphi \varepsilon \lambda \hat{\varsigma} \varsigma)$, pour la dévotion privée ou publique [...]. Certains de ces livres profitables étaient juste d'anciens récits apocryphes revisités pour les rendre salonfähig, comme nous le dirions en allemand - c'est-à-dire décents, acceptables, lisibles, politiquement corrects - si ce n'est à l'église, tout au moins dans le réfectoire des monastères ${ }^{181}$.

179 Ce point 2.2 résume et traduit quelques extraits d'un article étendu sur la question, avec l'autorisation de l'éditeur Brill que je remercie. Voir Clivaz, « Categories of Ancient Christian Texts ».

180 F. Bovon, «Beyond the Canonical and the Apocryphal Books, the Presence of a Third Category : The Books Useful for the Soul », HTR 105 (2012/2), p. 125-137 ; ici p. 135-136.

181 F. Bovon, « About Manuscripts and the Digital Era: A Personal Encounter », dans Reading Tomorrow. From Ancient Manuscripts to the Digital Era / Lire Demain. Des manuscrits antiques à l'ère digitale, C. Clivaz - J. Meizoz - F. Vallotton - J. Verheyden (éd.), avec B. Bertho, PPUR, 2012, ebook, p. 195-212 ; ici p. 206 : « I believe there was a third destiny: some books were neither rejected as apocryphal nor admitted as canonical, but were considered useful and profitable for the soul ( $\psi \cup \chi \omega \varphi \varepsilon \lambda \hat{\eta} \varsigma)$, for private or public devotion (...). Some of 
Bovon assume en outre de s'exprimer sur cette thématique depuis un cadre culturel religieux américain, qui ressemble souvent à un «match de tennis » entre évangéliques et libéraux, comme il l'explique avec humour ${ }^{182}$. Face à ce positionnement émique, Andrew Gregory a démontré clairement que, d'un point de vue strictement historique, il n'y avait aucune raison de ne pas considérer tous les textes chrétiens anciens comme utiles, pour une raison ou une autre, pour un groupe de chrétiens ou un autre ${ }^{183}$. La relecture de ces données antiques par les chercheurs contemporains s'avère donc être un lieu test intéressant de résistance du contexte religieux chrétien, en suivant la perspective adoptée par chacun. J'en prendrai encore deux exemples.

Premièrement, on peut comparer avec profit les deux préfaces des volumes de textes apocryphes chrétiens publiés dans la Pléiade. En 1997, François Bovon y évoquait certes la perspective historique de l'ouvrage, mais aussi son sentiment de transgression en publiant ces récits, laissant l'évaluation ultime aux lecteurs :

Il est paradoxal de composer un recueil d'écrits apocryphes chrétiens. Car qui dit recueil suggère aussitôt intérêt, valeur et autorité. En faisant un choix d'apocryphes pour la Bibliothèque de la Pléiade, les éditeurs ne confèrent-ils pas une certaine notoriété - voire une certaine autorité - à ce qui, par définition, doit en être privé ? Tout dépend finalement de l'attitude des lecteurs. Il leur revient d'approcher ces documents comme ils l'entendent: dans une perspective historique et littéraire - comme le sou-

those profitable books were just old apocryphal stories revisited to make them salonfähig, as we would say in German - that is, decent, acceptable, readable, politically correct - if not in church then at least in the refectory of monasteries ».

182 Bovon, «About Manuscripts », p. 125 : «I like tennis - both to play and to watch it. Nothing is more pleasant than watching an exchange between Federer and Nadal. There is a similar kind of exchange that has been going on in this country in recent years. On one side, there are evangelical New Testament scholars ; on the other, liberal scholars working on early Christianity ».

183 Merci à Andrew Gregory d'avoir mis à ma disposition cette citation d'un article à paraitre : "Yet it seems unlikely that any author set out to write a text that he or she did not think was 'useful for the soul', even if the gatekeepers of authority, or even few others, might have disagreed. As historians we may well be able to identify texts such as the Protevangelium of James, the Letter to the Laodiceans or 3 Corinthians that many early Christians may have considered useful for the soul. But as historians we might also want to say that the Gospel of Thomas, the Gospel of Judas and the Apocryphon of John were likely thought useful for the soul by some Christians, even if other Christians such as Irenaeus may have thought that these texts were to be rejected ». 
haiteraient les éditeurs -, heureux d'avoir enfin ces documents réunis et accessibles dans leur propre langue ${ }^{184}$.

Quelques années plus tard, en 2005, Jean-Daniel Kaestli écrivait en mémoire de Pierre Géoltrain la préface du second volume d'Ecrits apocryphes chrétiens. Le ton a évolué : dans les propos de Kaestli, on ne trouve pas trace d'un sentiment de transgression ou de paradoxe, mais au contraire une invitation au lecteur à reconsidérer la notion de Scriptura, pour la percevoir dans tous les textes du volume, placé sous le patronage de Priscillien :

Priscillien a défendu une conception large de l'Ecriture (Scriptura), incluant non seulement le recueil fermé des livres de la Bible (canon), mais encore un ensemble plus vaste et ouvert de textes extérieurs au canon, porteurs aussi du souffle de l'inspiration prophétique. Ne convient-il pas de donner raison à Priscillien et de reconnaître la commune appartenance des livres canoniques et apocryphes à une même Scriptura ? C'est peutêtre une des questions que la fréquentation de ces Ecrits apocryphes chrétiens fera naître chez le lecteur ${ }^{185}$.

Jean-Daniel Kaestli est ici proche du point de vue d'Andrew Gregory, et considère en outre que toute la littérature chrétienne est le fruit d'une inspiration, même si c'est davantage dans un sens poétique que religieux. Sa posture se tient au-delà des catégories canonique/apocryphe, lorsqu'il considère toute la production chrétienne comme «Ecriture », comme une Scriptura libre de tout contrôle ecclésial. Cette différence de posture entre Bovon et Kaestli, même à peu d'années de distance, signale une évolution culturelle plus globale, dont Bovon avait conscience : « le succès en France de la littérature apocryphe de la collection de la Pléiade signale probablement une extension de la sécularisation et le déclin du catholicisme romain dans ce pays $»^{186}$.

La métamorphose du titre de la célèbre collection allemande de publication des textes apocryphes chrétiens signale aussi l'évolution des catégories, un débat mené durant des décennies en dialogue et confrontation avec les travaux de l'association francophone du domaine l'Association pour l'étude de la littérature apocryphe chrétienne (AELAC). En 1983, Eric Junod a publié une importante étude sur cette question des catégories de texte, avec une définition

184 F. Bovon - P. Geoltrain (éd.), Ecrits apocryphes chrétiens I (Pléiade 442), Gallimard, 1997, p. IX.

185 J.-D. Kaestli - P. Geoltrain, «Introduction», dans Écrits apocryphes chrétiens II (Pléiade 516), P. Geoltrain - J.-D. Kaestli (éd.), 2005, p. XXI- XLI ; ici p. XLI.

186 Bovon, «Beyond the Canonical », p. 136. 
qui a fait date ${ }^{187}$. Mais il a fallu attendre trente ans pour que cette prise de position soit reconnue et ait un effet dans la recherche allemande. En 2012, Christoph Markschies a en effet souligné l'importance de cette définition dans son introduction au premier volume des Antike christliche Apokryphen ${ }^{188}$. Le titre précédent de cette série était Die neutestamentlichen Apokryphen ${ }^{189}$, soit une perspective où le Nouveau Testament était le point de référence.

Les débats menés à l'AELAC depuis les années 80 ont conduit à nommer les deux volumes de la Pléiade Ecrits apocryphes chrétiens, un label qui ne comportait déjà plus la référence au canon. Le choix de rebaptiser la série allemande Antike christliche Apokryphen atteste de lui-même de l'évolution des débats sur les catégories de textes chrétiens anciens. Ce qui surprend dans ces discussions ${ }^{190}$, c'est l'absence de réflexion sur l'influence du support matériel d'écriture sur la constitution des corpus et catégories. Mais à lire Eric Junod dans un article de 1992 au titre provocateur - «Apocryphes du Nouveau Testament »: une appellation erronée et une collection artificielle - , on se rend compte que tout est en place pour faire la jonction dans le débat entre cette problématique et celle de la matérialité de l'édition critique digitale :

Nous pensons en effet que la production d'apocryphes n'est limitée ni dans le temps ni dans l'espace, même si les conditions de production ont changé et continuent de changer selon les lieux et les temps. Et surtout,

187 E. Junod, « Apocryphes du NT ou apocryphes chrétiens anciens? Remarques sur la désignation d'un corpus et indications bibliographiques sur les instruments de travail récents », Etudes Théologiques et Religieuses 58 (1983), p. 409-421; ici p. 412 : «Voici donc la définition générale que nous proposerions des apocryphes chrétiens: textes anonymes ou pseudépigraphes d'origine chrétienne qui entretiennent un rapport avec les livres du NT et aussi de l'AT parce qu'ils sont consacrés à des événements racontés ou évoqués dans ces livres ou parce qu'ils sont consacrés à des événements qui se situent dans le prolongement d'événements racontés ou évoqués dans ces livres, parce qu'ils sont centrés sur des personnages apparaissant dans ces livres, parce que leur genre littéraire s'apparente à ceux d'écrits bibliques ».

188 C. Markschies, « Haupteinleitung », dans Antike christliche Apokryphen in deutscher Übersetzung, I. Band: Evangelien und Verwandtes, C. Markschies - J. Schröter - A. Heiser (éd.), Mohr Siebeck, 2012, p. 1-180 ; ici p. 112-114.

189 Pour une réponse de Wilhelm Schneemelcher à la définition d'Eric Junod, voir W. Schneemelcher (éd.), New Testament Apocrypha, R. McWilson (trans.), vol. 1, John Knox Press, 1992, p. 58-61.

190 Pour une synthèse approfondie de la problématique, on pourra se référer utilement à la préface de T. Burke - B. Landau (éd.), New Testament Apocrypha: More Noncanonical Scriptures, Eerdmans, 2016. 
nous voyons une contradiction entre l'idée même d'apocryphe, et l'idée de collection close ${ }^{191}$.

L'avis de Junod reflète typiquement une posture ancrée dans la culture imprimée : les conditions de production y sont minimisées au profit de l'impact des « idées », idée d'apocryphe ou idée de la collection close. Cette posture idéale sur la production de la pensée reflète ce que j'avais désigné en 2012 comme le point aveugle de la modernité, soit l'impact du support d'écriture et de production des contenus intellectuels sur la production des concepts et des idées ${ }^{192}$. A la lumière du point 2.1, nous pouvons ici très bien relire l'extension à l'œuvre dans la catégorie d'apocryphes - telle que souhaitée par Junod en 1983 et 1992 et telle que commencée à être mise en œuvre dans les séries Ecrits apocryphes chrétiens et les Antike christiliche Apokryphen - comme prête à s'insérer dans la transformation de la notion de corpus textuel à celle de collection digitale. Ce point dépendra bien sûr de la manière dont l'AELAC et les autres groupes de recherche concernés prendront le tournant de la culture digitale dans les années à venir, une question en cours ${ }^{193}$. En attendant, on peut faire œuvre utile en retraversant la problématique pour y observer l'importance de la matérialité du support d'écriture.

2.2 .2

La matérialité du support d'écriture et les catégories de textes chrétiens anciens

Pour prendre en compte la matérialité des supports d'écriture dans notre problématique, il faut commencer par constater que la catégorie dite «apocryphe » est née à un moment bien précis de l'histoire, sous la plume de J. A. Fabricius, même si elle s'appuie sur des éléments antérieurs. En effet, l'intérêt pour les apocryphes a été constant, mais pas toujours porté par les mêmes quêtes. C'est ce dont on se rend compte en comparant les points de vue de Fabricius et Lefèvres d'Etaples, deux siècles plus tôt.

Dans sa préface au corpus des apocryphes, J. A. Fabricius commente ainsi leur utilité : ces livres peuvent être lus non seulement sans désavantage, mais « cum utilitate quoque, si idoneus lector accesserit » ${ }^{194}$, ce que Gérard Poupon

191 E. Junod, « 'Apocryphes du Nouveau Testament': Une appellation erronée et une collection artificielle », Apocrypha 3 (1992), p. 17-46 ; ici p. 37.

192 Clivaz, « Common Era », p. 29-35, part. p. 35 .

193 Voir par exemple le projet d'édition conduit par Frédéric Amsler avec un subside du Fonds National Suisse, Editer la littérature apocryphe chrétienne, 2017-2021, <http://p3.snf. ch/Project-157961>.

194 J. A. Fabricius, Codex apocryphus Novi Testamenti, Vol. 1. Hambourg, Sumptib. Benjam. Schiller, $1719^{2}$, f. $7 \mathrm{r}$; cité par Junod, « 'Apocryphes du Nouveau Testament' », p. 22, note 11. 
traduit par «rendre service aux personnes instruites » ${ }^{195}$. L'utilitas mentionnée ici n'est certainement pas une « utilité pour l'âme », mais une amélioration intellectuelle pour le idoneus lector. Ce contraste apparaît encore plus clairement à comparer Fabricius avec Lefèvre d'Etaples qui reste, pour sa part, intéressé à «la piété » de ces textes qui pour lui « respirent le Christ à chaque page »196. Une attitude similaire de piété se retrouve chez le luthérien Nerbel et d'autres ${ }^{197}$. Autrement dit, en deux siècles, l'utile à l'âme de Lefèvres d'Etaples et Nerbel est devenu chez Fabricius, dans l'antichambre des Lumières, un «utile pour la quête intellectuelle».

Il est intéressant de relever que cette période correspond également à un tournant dans les études bibliques, avec, en 1711, l'élaboration par Mastricht des 43 critères pour juger d'une variante textuelle ou en 1725 , celle des canons de Bengels ${ }^{198}$. Au moment où Fabricius rédige son canon des apocryphes, Robert Lowth, professeur de poésie à l'université d'Oxford, enseigne la première lecture littéraire et poétique de la Bible ${ }^{199}$. Ce tournant épistémologique de l'Age Classique, si finement décrit par Foucault ${ }^{200}$, souligne bien qu'on ne peut pas établir de catégorie atemporelle pour décrire les textes chrétiens anciens : le regard porté sur ces textes est inévitablement empreint du contexte culturel religieux, comme nous l'avons vu au point 2.2.1 pour notre contexte contemporain.

Les utilisateurs de ces textes, du quidam au chercheur, se retrouvent en revanche à travers les siècles à les grouper, les regrouper, en faire des listes et des ensembles. C'est cet aspect qui est au bout du compte observable et descriptible. Le défi est d'adjoindre ici l'analyse des supports matériels d'écriture, telle que promue par Chartier notamment ${ }^{201}$, à l'analyse historique de Foucault. De

195 G. Poupon, «Les Actes apocryphes des Apôtres de Lefèvre à Fabricius », dans Les Actes apocryphes des apôtres: christianisme et monde païen, F. Bovon (éd.), Labor et fides, 1981, p. $25-47$; ici p. 43.

196 I. D. Backus, «Renaissance Attitudes to New Testament Apocryphal Writings: Jacques Lefèvre d'Etaples and His Epigones », Renaissance Quarterly 51 (1998), p. 1169-1198 ; ici p. 1174-1175.

197 Backus, « Renaissance Attitudes », p. 1189.

198 E. Epp, «Issues in New Testament Textual Criticism. Moving from the Nineteenth Century to the Twenty-First Century », dans Rethinking New Testament Textual Criticism, D. A. Black (éd.), Baker Academic, 2002, p. 17-76 ; ici p. 21.

199 Z. Konstantinovic, « Die Nachwirkungen der Bibel als Problem der Vergleichenden Literaturwissenschaft », dans Die Bibel im Verständnis der Gegenwartsliteratur, J. Holzner U. Zeilinger (éd.), Verlag Niederösterreichisches Pressehaus, 1988, p. 17-24 ; ici p. 17.

200 Foucault, Les mots et les choses. Pour une présentation complète de cette analyse, voir Clivaz, « Categories of Ancient Texts », p. 57-58.

201 Chartier, Les métamorphoses, p. 12-14; Clivaz, «The New Testament at the Time of the Egyptian Papyri », p. 25-27. 
fait, en observant l'émergence de nouveaux corpus en ligne - qui deviendront peut-être bientôt des collections multimodales -, on achève de se convaincre de l'intérêt de cette double lecture. En effet, il suffit de regarder par exemple l'évolution du matériel présenté dans les collections du site Center for the Study of the New Testament Manuscripts (CSNTM). Premièrement, depuis plusieurs années, le site met à disposition des manuscrits du Nouveau Testament avant qu'ils n'aient reçu leur classification Gregogy-Aland par l'INTF, contribuant ainsi à une certaine dérégulation du système international de repérage de ces manuscrits. Deuxièmement, de plus en plus de textes non néotestamentaires prennent place dans les manuscrits mis à disposition, y compris des textes apocryphes chrétiens ${ }^{202}$.

Autrement dit, nous retrouvons ici un trait propre à la culture de l'édition digitale, déjà souligné dans ce volume : le tournant du texte au document. En effet, à partir du moment où l'objet d'étude contient différents types de textes, canoniques et apocryphes, la logique du document qu'on voit finit par l'emporter inexorablement. On a déjà vu que dans le projet saws, les délimitations de contenu étaient absolument ouvertes; de même, le projet Paratexts of the Bible signale dans sa présentation même un périmètre d'extension plus qu'appréciable 203 .

Jusqu'où l'arrivée de collections digitales peut-elle ouvrir le champ ? On a vu avec Mombert qu'il y avait à tout projet des limites de ressources naturelles en temps et en argent. En même temps, la littérature arabe connaît un exemple extrême de bibliothèque ouverte avec la base de données Shamela, téléchargeable librement. Structurée selon un index thématique général, elle contient des dizaines de milliers d'ouvrages ${ }^{204}$. Si on a là un exemple particulier, il permet toutefois de renouveler une observation souvent faite jusqu'ici : les délimitations sont aussi posées par les communautés, les groupes qui s'intéressent à un objet digital. Autrement dit, si Shamela existe, c'est qu'il y a un public vaste - d'utilisateurs qui la valide comme telle et la visite.

C'est d'un côté une vérité de La Palisse, mais d'un autre, c'est la révolution que nous avons vu proposée par Thoutenhoofd : adjoindre l'analyse des

202 Centre for the Study of New Testament Manuscripts, <http://www.csntm.org/>. Exemple: le Chester Beatty CBL BP XVI, avec l'Apocryphon of Jannes and Jambres the Magicians.

203 ParaTextBib, <http://paratexbib.eu/>. The Paratexts of the Bible project est décrit ainsi: «The project aims at publishing an online e-Clavis of all relevant biblical paratexts in Greek and their manuscript witnesses. Some 200 of these paratexts will be critically edited and published in a printed book. The main results will be synthesized in a monograph. This will require registering and ordering more than 2500 manuscripts of the Old and New Testament. More than 500 paratexts will be studied ».

Assef, « Bibilothèque numérique "Shamela" ». 
sciences sociales - users inclus - à celle de la philologie ${ }^{205}$. De ce point de vue, on peut tabler sur un nombre certainement encore conséquent de groupes et communautés qui garderont un intérêt centré sur les textes canoniques bibliques, comme nous l'avons souvent observé dans l'analyse de ce chapitre. Quels seront les liens à venir de ces groupes et communautés à l'ensemble de la recherche académique : ce point reste ouvert, et nous dira, de par sa fluctuation si nous restons encore dans une ère culturelle chrétienne ou non.

La question est sans doute plus ouverte pour le devenir des corpus d'ensemble de textes chrétiens anciens, maintenus par de simples cercles académiques avec peu de support sociologique au sens large : ils se retrouvent de plus en plus insérés dans des collections digitales larges, telles le Thesaurus Linguae Graecae ${ }^{206}$ par exemple, et plus le temps passe, moins il semble y avoir de raison pour ne pas les mettre en réseaux avec d'autres textes antiques. C'est la sortie du corps textuel qui s'annonce, un bouleversement qui demande qu'au point suivant, nous revisitions brièvement ce lien séculaire des textes au corpus et au corps, avant d'affronter, pour conclure, la transformation qui va reconfigurer la publication de toutes les collections digitales, la multimodalité. Je proposerai que la multimodalité sera l'un des lieux privilégiés de recomposition du rapport au corps pour les expressions culturelles digitales, Ecritures bibliques incluses.

\subsection{Corps, textes et multimodalité digitale}

2.3.1 Le corps et le texte, une longue histoire culturelle

La question de l'accord, harmonieux ou non, entre la matérialité du support d'écriture et le corps n'a cessé de traverser la mémoire culturelle occidentale, comme le souligne Emmanuèle Baumgartner dans un article déjà signaléen ${ }^{207}$ $\mathrm{Au}$ cours de ce livre, nous en avons croisé plusieurs exemples, notamment en lien à l'héritage culturel chrétien. Au chapitre 1, nous avons vu que Lyotard, dans son ouvrage posthume, reprenait Ap 6,14 et Augustin pour comparer la couverture d'un volume à de la peau ${ }^{208}$. Au chapitre 2 , nous avons vu que le terme humanité avait pu désigner un «moule de chair», le ventre de la femme ; cet usage sera transposé plus tard pour désigner le fait d'imprimer comme « mettre en moule $»^{209}$.

Au chapitre 3, nous avons vu que la génomique utilisait l'expression « décoder le livre de vie » pour décrire sa tâche, sans toutefois prendre acte des échos

205 Thoutenhoofd, « Presence beyond digital philology », p. 285 ; cité p. 182 ci-dessus.

206 Thesaurus Linguae Graecae, <http://stephanus.tlg.uci.edu/>.

207 Baumgartner, « Du manuscrit retrouvé au corps retrouvé ».

208 Lyotard, La Confession d'Augustin, p. 59 ; cité au chapitre 1, p. 28.

209 Huguet, «Humanité », Le dictionnaire de référence, <https://classiques-garnier.com/hu guet-dictionnaire-du-xvie-s.html> ; cité au chapitre 2, p. 57 . 
divers de cette expression parfois ambiguë dans l'Apocalypse biblique (Ap 3, 5 et 20,15$)^{210}$. Le Codex Sinaiticus, cité plus haut dans ce chapitre, offre un rapport au vivant particulier : on peut à plusieurs endroits y repérer les pores de la peau de l'animal ou même l'une de ses veines ${ }^{211}$.

On pourrait ajouter de nombreux exemples, tous plus parlants les uns que les autres. Ainsi cette bandelette de momie chrétienne sur laquelle on a écrit une phrase très proche du logion 5 de l'Evangile apocryphe de Thomas ${ }^{212}$. Ou encore l'histoire hagiographique de Mélanie la Jeune (4e siècle de notre ère), qui raconte qu'après avoir frôlé la mort à son second accouchement, elle obtient de son mari le droit à la survie par l'abstinence. Elle se met alors à écrire des livres qu'elle qualifiera de «petits corps », somatioi, et qui se substituent ainsi à la procréation ${ }^{213}$. Par ailleurs, on a cherché à limiter le livre dans le christianisme naissant, en se méfiant d'une écriture surabondante (Jn 21, 25), ou à faire gagner le corps sur le livre, quitte à le manger (Ap 10, 10).

Dans un article de 2015, j'ai mené une enquête complète sur le vocabulaire du rouleau et du livre dans le Nouveau Testament ${ }^{214}$, qui offre parfois des liens au corps très concrets, comme ce passage de l'Épître aux Hébreux où Moïse asperge de sang le livre, et cela même avant le peuple : «Et il aspergea le livre lui-même et tout le peuple en disant : Ceci est le sang de l'alliance que Dieu a prescrite pour vous » (He 9, 19-20). De fait, c'est bien du rouleau dont il est question dans ce corpus de texte, et non pas du codex, du livre de pages : le «livre de vie» est un «rouleau de vie ». On se retrouve donc devant une constatation surprenante : alors que le christianisme a tant été assimilé à une religion du livre, c'est bien le rouleau qui est le support matériel d'écriture représenté dans les vingt-sept livres du Nouveau Testament.

La prise en compte de cette divergence dans les représentations devrait nous rendre d'autant plus attentifs à situer historiquement l'idée de «religion du livre ». De fait, cette expression n'apparait dans le vocabulaire culturel occidental qu'en deuxième moitié $d u 19^{\mathrm{e}}$ siècle $^{215}$, et n'est pas à ne pas confondre

$210 \quad$ Voir chapitre 3, p. 150.

211 Par exemple en haut du folio 113 r. (Psaumes 89,16 à 92, 1) <http://www.codexsinaiticus. $\mathrm{org} / \mathrm{en} /$ manuscript.aspx ?dir $=$ next\&folioNo $=1 \&$ lid $=$ en\&quireNo $=62 \&$ side $=\mathrm{v} \& z$ zoomSli der $=0>$.

A. Luijendijk, «'Jesus says: 'There Is Nothing Buried That Will Not Be Raised'. A LateAntique Shroud with Gospel of Thomas Logion 5 in Context », ZAC 15/3 (2011), p. 389-410. D. Gorce (éd.) Vie de Sainte Mélanie (SC 9o), Cerf, 1962, § 23 et 36.

214 C. Clivaz, «Pratiques de lecture, identités et prise de conscience : la question des miniatures et du BIBLARIDION d'Apocalypse 10,2.9-10 ", dans Les judaïsmes dans tous leurs états aux I ${ }^{\text {er-III }}$ siècles (Les Judéens des synagogues, les chrétiens et les rabbins). Actes du colloque de Lausanne 12-14 décembre 2012, JAOC 5, C. Clivaz - S. Mimouni - B. Pouderon (éd.), Brepols, 2015, p. 321-344 ; ici p. 323-324.

215 Voir par exemple F. H. Geffcken, Staat und Kirche, in ihrem Verhältniss geschichtlich entwickelt, Berlin, 1875, p. 225. 
avec l'expression coranique des « gens du Livre », contrairement à ce qu'a affirmé par exemple Wilfred Cantwell Smith ${ }^{216}$. Dans son article, Smith ne fait aucune mention de ce qui est toujours sous-estimé dans la recherche : la différence sémantique entre les ahl al-Kitab du Coran et les « religions du Livre ». Pourtant, il signale la conférence programmatique donnée à Londres en 1870 par Friedrich Max Müller, Sacred Books of the East ${ }^{217}$, qui peut être considérée comme le facteur du succès de l'appellation « religion du Livre ». Max Müller propose une classification complète de huit religions à partir de la notion de «livre » :

Avec ces huit religions, la bibliothèque des Livres Sacrés de toute la race humaine est complète, et une étude attentive de ces huit codes, écrits en sanskrit, pâli, zen, hébreu, grec et arabe et finalement en chinois, pourrait en elle-même ne pas sembler démesurée pour un seul chercheur ${ }^{218}$.

On fait face ici à un point de vue typique de l'académisme du 19e siècle triomphant : un point de vue universalisant, la possibilité de tout résoudre par un système et l'exaltation de la figure du chercheur en sciences humaines solitaire. Ce constat ne surprend pas non plus au regard du fait que le statut juridique du livre et de l'auteur a été fixé définitivement en $185^{219}$ : désormais le texte existe, telle une entité à part entière. Le point de vue de Max Müller fera mouche, notamment parmi les théologiens protestants, tels Friedrich Heinrich Geffcken ou Carl Theodor A. Liebner, qui relieront de manière anachronique la doctrine de Calvin à l'idée de la Buchreligion ${ }^{220}$. Sur la base de ces observations, on comprend que le contexte de la culture imprimée à son apogée a favorisé, dans les milieu théologiques protestants allemands en

216 W. C. Smith, «Scripture as form and concept : their emergence for the Western world», dans Rethinking Scripture: Essays from a Comparative Perspective, W. C. Smith, New York University Press p. $29-57$; ici p. 30 : «It is illuminating, I suggest, to begin with the seventh century ad as the virtually culminating stage of the process, and to trace it then backwards in time ».

217 Müller, «Second Lecture Delivered at the Royal Institution »; cite par Smith, « Scripture as form and concept», p. 30 et 33 .

218 Müller, «Second Lecture Delivered at the Royal Institution », p. 56 : «With these eight religions the library of the Sacred Books of the whole human race is complete, and an accurate study of these eight codes, written in Sanskrit, Pâli, and Zend, in Hebrew, Greek, and Arabic, lastly in Chinese, might in itself not seem too formidable an undertaking for a single scholar».

219 R. Chartier, Culture écrite et société. L'ordre des livres (XVI ${ }^{e}-X V I I I^{e}$ siècles), Albin Michel, 1996, p. 5 .

220 Geffcken, Staat und Kirche, p. 225 ; C. T. A. Liebner et alii (éd.), Jahrbücher für deutsche Theologie $17(1872 / 1)$, p. 93 . 
particulier, l'adoption de l'idée de « religion du livre » dans la deuxième moitié du 19e siècle.

Ce parcours historique permet de situer le moment d'entrée en force du concept de « religion du livre », avec son lien net à la culture imprimée, souligné par Jean-Claude Carrière ${ }^{221}$. Il dégage en contrecoup la possibilité de penser différemment le lien du christianisme aux médias, et en particulier aux supports d'écriture digitale. On ne peut dès lors qu'approuver le rapport Sola lectura? de la SEK qui affirme clairement « das Christentum ist keine Buchreligion $»^{222}$. En même temps, au vu des nombreux liens rappelés dans ce point 2.3.1 entre corps et textes, et cela dans la veine même de l'héritage chrétien, l'impact symbolique de la sortie du livre, de la sortie du monde des corpus textuels ne peut pas se faire sans profonds bouleversements : on l'a largement vu avec Jacques Derrida ${ }^{223}$.

$\mathrm{Au}$ sein des transformations en cours, le point suivant propose, pour conclure le parcours de ce chapitre 4, de considérer que les Ecritures digitales ont la possibilité d'explorer et d'habiter une nouvelle modalité de relation au corps, via leurs expressions numériques multimodales.

2.3 .2

Ecritures digitales et multimodalité : nouer un nouveau rapport au corps

Faire face à la sortie du corpus textuel sera un travail de longue haleine. On a ici plus que jamais besoin de réserves métaphoriques pour penser ce tournant. $\mathrm{Au}$ point 1.3, nous avons évoqué les différentes métaphores disponibles pour penser les relations intertextuelles, par-delà l'arbre du stemma. J'ai proposé qu'on y réfléchisse également avec le blob, associé au mycélium, en raison de sa capacité à transmettre observable, mais non encore expliquée ${ }^{224}$. Parce que le blob transmet autrement, il représente une réserve métaphorique intéressante pour dire que nous voyons des processus de transmission textuels et oraux à l'œuvre entre différents objets culturels, mais sans arriver forcément à en dire la causalité. Il nous invite à respecter le temps de l'observation, avant d'aller à celui des conclusions de causalité, soit un changement épistémologique d'importance pour les sciences humaines.

La quête de la métaphore, de la réserve d'image et d'imaginaire, est certainement aussi nécessaire pour penser le rapport corps-texte hors du corpus textuel. L'art peut nous offrir ici des chemins inattendus, pour penser de nouvelles

\footnotetext{
221 Carrière - Eco, N'espérez pas, édition Kindle, l. 1290-1298.

222 SEK, Sola lectura?, p. 7.

223 Voir l'Introduction, p. 3-4 et chapitre 1, p. 28.

224 CNRS, «Le 'blob' capable d'apprendre... », <http://www2.cnrs.fr/presse/communi que/4837.htm > ; cité en p. 191 ci-dessus.
} 
relations entre les textes, l'informatique et le vivant. La performance artistique Genesis d'Edouardo Kac, menée en 1999, est à cet égard exemplaire :

Kac produit un gène synthétique en traduisant en code morse une phrase tirée du livre de la Genèse puis en convertissant ce code en paires de bases d'ADN, conformément à un principe de conversion élaboré expressément pour le projet. Le gène a ensuite été exprimé dans la bactérie E. Coli. Par le truchement d'Internet, les participants pouvaient allumer une boîte d'éclairage ultraviolet dans la galerie et faire muter la bactérie. Le tout se trouvait dans une enceinte de protection contre les ultraviolets, rendant ainsi la bactérie inoffensive pour les visiteurs de la galerie ${ }^{225}$.

Après le temps de l'exposition, l'ADN synthétisé a été retransformé en code $\mathrm{ADN}$, puis code morse, puis verset biblique : la bactérie avait légèrement transformé le texte, comme on peut le voir en ligne, avec les trois versions aller et retour $^{226}$. Il s'agit en quelque sorte d'une anticipation artistique des tests de stockage d'information dans de l'ADN, conduits depuis 2012, comme on l'a $\mathrm{vu}^{227}$. Ce texte transformé par le vivant - une bactérie - est une représentation forte des nouveaux liens que les mots écrits peuvent contracter avec le vivant via l'informatique. Le texte utilisé se trouve qui plus est être Gn 1, 26, qui implique la domination d'Adam sur les animaux : on appréciera l'ironie de voir ce texte précisément transformé par un organisme vivant.

Une telle expérience provoque aussi un sentiment d'étrangeté, les repères sont bousculés. La culture imprimée nous avait fait intégrer, à un point bien plus fort que ce que nous pouvions imaginer, le sentiment de sécurité devant le texte stable et les textes assemblés en corpus: cette époque est révolue. A ce bouleversement, au sortir du corpus textuel, s'ajoute encore ce point que Sarah Mombert considère comme l'un des plus importants de la transition vers la collection digitale : la possibilité de lier textes et images ${ }^{228}$, que j'ai étendue au point 2.1 à la thématique de la multimodalité, paramètres sonores inclus. En finale de cet essai, je propose de comprendre que c'est notamment via la multimodalité des objets digitaux, rassemblés en collections, que l'écriture digitale va participer à l'émergence de cette nouvelle relation entre le corps de l'homme et les machines, entre le corps de l'homme et les technologies.

225 Eduardo Kac. Genesis 2, <http://www.fondation-langlois.org/html/f/page.php?NumPage= 278> ; Eduardo Kac, Genesis, <http://www.ekac.org/genesis.html>; Eduardo Kac. Genesis short description, <http://www.ekac.org/gensumm.html >.

226 Eduardo Kac. Work on the Genesis Series 2001, <http://www.ekac.org/genseries.html>.

227 Voir chapitre 3, p. 131.

228 Mombert, « From Books to Collections », édition Kindle, l. 5157 ; cité p. 201 ci-dessus. 
Les Ecritures digitales contribuent pour leur part à ce processus et permettent d'explorer le rapport au donné biblique - corps dans de nouvelles formes, pardelà l'enclos rassurant du corpus textuel.

Cette proposition se base premièrement sur toute l'enquête conduite au chapitre 3 sur la multimodalité. Nous avons vu, avec l'exemple de la vidéo YouTube d'Emmanuelle Riva ${ }^{229}$, que la présence de la voix et de la corporéité de l'actrice permettait la porosité de son «je » au cours de l'interview ${ }^{230}$. Attentifs aux travaux d'Henri Meschonnic, nous avons vu que la présence de l'oralité dans le discours culturel numérique provoquait de multiples transformations, y compris dans l'émergence de nombreuses formes de performances littéraires qui remettent au premier plan le corps de l'écrivain ${ }^{231}$. Comme je l'ai synthétisé au début du point consacré à ce thème au chapitre 3, la multimodalité «provoque l'écriture digitale qui devient ainsi un processus global qui sort d'une définition liée au texte à proprement parler $»^{232}$.

Les outils de publication multimodale tels Scalar, les eTalks ou les Lab du journal Le Temps, font une place décisive et remarquée à la corporéité dans l'acte éditorial même ${ }^{233}$. Voix, visages, timbres, sons : tout est mis en place pour rendre sa place au corps et le joindre enfin aux mots des textes, qui ne peuvent plus prétexter de la réserve d'usage acquise via le papier silencieux. Sans oublier que le CLI, et donc les signes écrits, garde la maîtrise ultime sur le code informatique ${ }^{234}$, voici l'écriture digitale désormais embedded - enclose, incorporée, encastrée, encapsulée, insérée - dans la matière digitale, et par là même, elle retrouve le corps, elle est redonnée au corps, jusqu’à acquérir la capacité d'être accueillie dans un code génétique qui vient doubler le code informatique.

Cette aventure d'une nouvelle relation au corps, les Ecritures digitales ont déjà commencé à l'expérimenter dans les liens qu'elles contractent avec les sons et les images, chaque jour davantage. La représentation la plus parlante de cette mutation est certainement le pompier Montag, dans le roman Fahrenheit 451, qui finit par devenir des chapitres de l'Ecclésiaste et de l'Apocalypse :

«Tu veux nous rejoindre Montag?».

«Oui ».

\footnotetext{
229 «Emmanuelle Riva, interview 1959», <https://www.youtube.com/watch?v=kRMaDYU hos4>.

230 Voir chapitre 3, p. 118-119.

231 Voir chapitre 3, p. 119.

232 Voir chapitre 3, p. 139.

233 Voir chapitre 3, p. 140-141.

234 Voir chapitre 3, p. 144-145.
} 
«Qu'as-tu à offrir?».

« Rien. Je pensais que j'avais une partie du livre de l'Ecclésiaste et peutêtre un peu de l'Apocalypse, mais je n'ai même pas cela maintenant ». «Le livre de l'Ecclésiaste serait parfait. Où est-il ? »

«Ici ». Montag toucha sa tête ${ }^{235}$.

Accepter de joindre les Ecritures dans la culture digitale entraîne forcément à retrouver un contact plus oral et imagé à ces textes millénaires. Pour les membres des communautés chrétiennes, elles leur offrent le défi de (re)devenir des Human Libraries, corpus textuels vivants : après tout, dans l'Antiquité c'est bien ce qui advenait, puisque l'immense majorité des personnes ne savait pas lire. Après tout, c'est bien ce que Karl Barth avait à l'esprit en rappelant avoir rédigé son commentaire sur l'Epître aux Romains pour rejoindre une unsichtbare Gemeinschaft, et non pas pour publier un livre ${ }^{236}$. A l'image de l'alternative offerte par Montag entre livre de l'Ecclésiaste et de l'Apocalypse, je dirais que le regard à porter sur cette évolution est ouvert, entre chemin de sagesse réaliste à la mode de l'Ecclésiaste et sentiment de catastrophe aux couleurs de l'Apocalypse.

Tous les chercheurs en sciences bibliques traversent ce type d'hésitations. Pete Phillips vient de publier un article interrogeant la fragilité actuelle du texte, mais conclut que la culture visuelle permet plutôt de « magnifier » la voix textuelle ${ }^{237}$. Je me suis moi-même demandé en 2014 si le Nouveau Testament deviendrait un tout petit livre perdu sur internet ${ }^{238}$. Je conclus aujourd'hui cette enquête en tournant mon regard vers les possibles émergents, ouverts et risqués, pour ces Ecritures digitales, appelées à contracter de nouveaux liens avec la corporéité, dans leurs expressions multimodales. Tel est le fruit à cueillir sur l'arbre numérique.

235 Bradbury, Fahrenheit 451, p. 142 ; cité au chapitre 3, p. 131.

236 Voir chapitre 1, p. 22.

237 P. Phillips, «The Power of Visual Culture and The Fragility of the Text», dans D. Hamidović - C. Clivaz - S. Bowen Savant (éd.), Ancient Manuscripts in Digital Culture (DBS 3), Brill, 2019, p. 30-49; ici p. 46 : «Contrary to the suggestion in the title of this paper, visual culture seems to magnify the voice of the text rather than to minimize it. In each of the examples, we see a kind of symbiosis between the Biblical text and visual culture in which each seeks strength from the other, and each seeks to make its communicative task complete through the other».

238 Clivaz, « New Testament in a Digital Culture : A Bibliaridion (Little Book) Lost in the Web?». 